\title{
An anisotropic distribution of spin vectors in asteroid families ${ }^{\star}$
}

\author{
J. Hanuš ${ }^{1}$, M. Brož ${ }^{1}$, J. Ďurech ${ }^{1}$, B. D. Warner ${ }^{2}$, J. Brinsfield ${ }^{3}$, R. Durkee ${ }^{4}$, D. Higgins ${ }^{5}$, R. A. Koff ${ }^{6}$, J. Oey ${ }^{7}$, \\ F. Pilcher ${ }^{8}$, R. Stephens ${ }^{9}$, L. P. Strabla ${ }^{10}$, Q. Ulisse ${ }^{10}$, and R. Girelli ${ }^{10}$ \\ 1 Astronomical Institute, Faculty of Mathematics and Physics, Charles University in Prague, V Holešovičkách 2, 18000 Prague, \\ Czech Republic \\ e-mail: hanus.home@gmail.com \\ 2 Palmer Divide Observatory, 17995 Bakers Farm Rd., Colorado Springs, CO 80908, USA \\ 3 Via Capote Observatory, Thousand Oaks, CA 91320, USA \\ ${ }^{4}$ Shed of Science Observatory, 5213 Washburn Ave. S, Minneapolis, MN 55410, USA \\ 5 Hunters Hill Observatory, 7 Mawalan Street, ACT 2913 Ngunnawal, Australia \\ 6980 Antelope Drive West, Bennett, CO 80102, USA \\ 7 Kingsgrove, NSW, Australia \\ 84438 Organ Mesa Loop, Las Cruces, NM 88011, USA \\ 9 Center for Solar System Studies, 9302 Pittsburgh Ave, Suite 105, Rancho Cucamonga, CA 91730, USA \\ 10 Observatory of Bassano Bresciano, via San Michele 4, Bassano Bresciano (BS), 25020 Brescia, Italy
}

Received 30 May 2013 / Accepted 16 September 2013

\section{ABSTRACT}

Context. The current number of $\sim 500$ asteroid models derived from the disk-integrated photometry by the lightcurve inversion method allows us to study the spin-vector properties of not only the whole population of main-belt asteroids, but also of several individual collisional families.

Aims. We create a data set of 152 asteroids that were identified by the hierarchical clustering method (HCM) as members of ten collisional families, among which are 31 newly derived unique models and 24 new models with well-constrained pole-ecliptic latitudes of the spin axes. The remaining models are adopted from the DAMIT database or a few individual publications.

Methods. We revised the preliminary family membership identification by the HCM according to several additional criteria: taxonomic type, color, albedo, maximum Yarkovsky semi-major axis drift, and the consistency with the size-frequency distribution of each family, and consequently we remove interlopers. We then present the spin-vector distributions for asteroidal families Flora, Koronis, Eos, Eunomia, Phocaea, Themis, Maria, and Alauda. We use a combined orbital- and spin-evolution model to explain the observed spin-vector properties of objects among collisional families.

Results. In general, for studied families we observe similar trends in $\left(a_{\mathrm{p}}, \beta\right)$ space (proper semi-major axis vs. ecliptic latitude of the spin axis): (i) larger asteroids are situated in the proximity of the center of the family; (ii) asteroids with $\beta>0^{\circ}$ are usually found to the right of the family center; (iii) on the other hand, asteroids with $\beta<0^{\circ}$ to the left of the center; (iv) the majority of asteroids have large pole-ecliptic latitudes $\left(|\beta| \gtrsim 30^{\circ}\right)$; and finally (v) some families have a statistically significant excess of asteroids with $\beta>0^{\circ}$ or $\beta<0^{\circ}$. Our numerical simulation of the long-term evolution of a collisional family is capable of reproducing the observed spinvector properties well. Using this simulation, we also independently constrain the age of families Flora ( $1.0 \pm 0.5 \mathrm{Gyr})$ and Koronis (2.5-4 Gyr).

Key words. methods: observational - minor planets, asteroids: general - techniques: photometric - methods: numerical

\section{Introduction}

An analysis of rotational state solutions for main belt asteroids has been performed by many authors. All these authors observed the deficiency of poles close to the ecliptic plane (e.g., Magnusson 1986; Drummond et al. 1988; Pravec et al. 2002; Skoglöv \& Erikson 2002; Kryszczyńska et al. 2007). Hanuš et al. (2011) showed that this depopulation of spin vectors mainly concerns smaller asteroids $(D \lesssim 40 \mathrm{~km})$, while the larger asteroids $(60 \lessgtr D \lesssim 130-150$ km, Kryszczyńska et al. 2007; Paolicchi \& Kryszczyńska 2012) have a statistically significant excess of prograde rotators, but no evident lack of poles close to the ecliptic plane. The observed anisotropy of pole vectors of smaller asteroids is now believed to be a result of YORP thermal

* Tables 3-5 are available in electronic form at http://www . aanda.org torques $^{1}$, and of collisions that systematically evolve the spin axes away from the ecliptic plane. The prograde excess of larger asteroids is probably caused by a primordial preference that agrees with the theoretical work of Johansen \& Lacerda (2010). While the number of asteroids with known rotational states grows, we can not only study the spin vector distribution in the whole main-belt asteroids (MBAs) or near-Earth asteroids (NEAs) populations, but we can also focus on individual groups of asteroids within these populations, particularly on collisional families (i.e., clusters of asteroids with similar proper orbital elements and often spectra that were formed by catastrophic breakups of parent bodies or cratering events).

1 Yarkovsky-O'Keefe-Radzievskii-Paddack effect, a torque caused by the recoil force due to anisotropic thermal emission, which can alter both rotational periods and orientation of spin axes, see e.g., Rubincam (2000). 

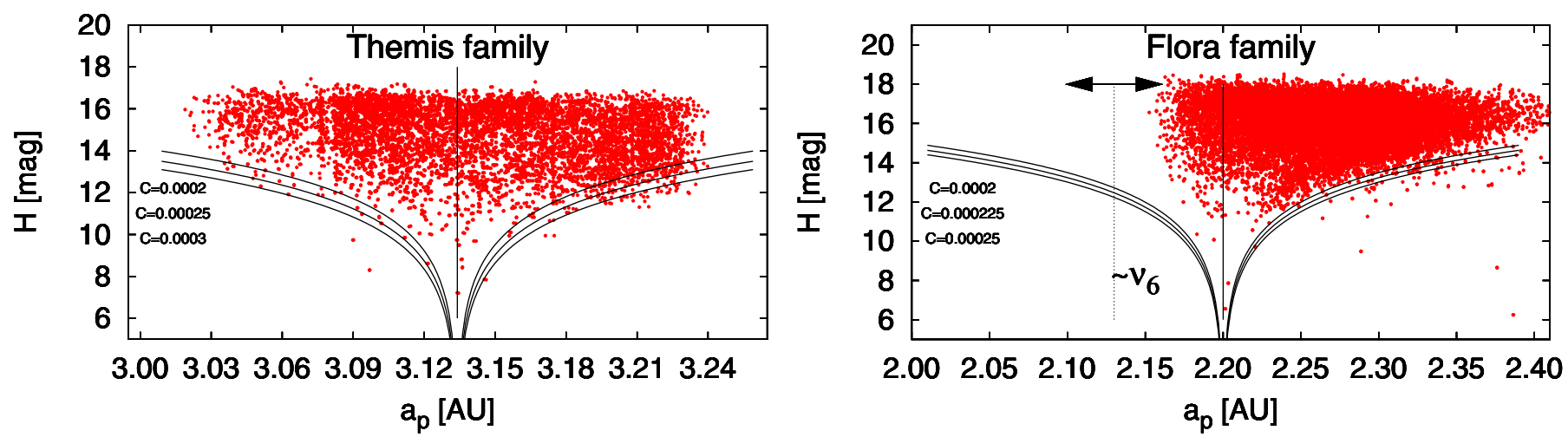

Fig. 1. Dependence of the absolute magnitude $H$ on the proper semi-major axis $a_{\mathrm{p}}$ for the Themis family (left) and for the Flora family (right) with the likely positions of the family centers (vertical lines). We also plot three $\left(a_{\mathrm{p}}, H\right)$ borders of the family for different parameters $C$ (different values correspond to a different initial extent of the family or different age and magnitude of the Yarkovsky semi-major axis drift) by gray lines, the optimal border corresponds to the middle line. The vertical dotted line represents the approximate position of the secular $v_{6}$ resonance for the inclination typical for Flora family members and the horizontal arrow its approximate range.

The theory of dynamical evolution of asteroid families (e.g., Bottke et al. 2006) suggests that the Yarkovsky ${ }^{2} /$ YORP effects change orbital parameters of smaller asteroids $(\$ 30-50 \mathrm{~km})-$ the semi-major axis of prograde rotators slowly grows the course of time, unlike retrograde rotators whose semi-major axis decreases. This phenomenon is particularly visible when we plot the dependence of the absolute magnitude $H$ on the proper semimajor axis $a_{\mathrm{p}}$ (see an example of such a plot for Themis family in Fig. 1, left panel). In addition, various resonances (e.g., meanmotion resonances with Jupiter or Mars, or secular resonances) can intersect the family and cause a decrease in the number of asteroids in the family by inducing moderate oscillations to their orbital elements (Bottke et al. 2001) as can be seen in Fig. 1 for the Flora family, where the secular $v_{6}$ resonance with Saturn almost completely eliminated objects to the left of the center of the family. The $v_{6}$ resonance has its center at $2.13 \mathrm{AU}$ for objects with $\sin I \sim 0.09$, which is typical of Flora family members. It develops objects which then approach the proximity of the resonance. Some resonances can, for example, capture some asteroids on particular semi-major axes (Nesvorný \& Morbidelli 1998).

Laboratory experiments strongly suggest that a collisionallyborn cluster should initially have a rotational frequency distribution close to Maxwellian (Giblin et al. 1998) and an isotropic spin vector distribution. For several families, we already know their age estimates (e.g., $2.5 \pm 1.0 \mathrm{Gyr}$ for Koronis family, Bottke et al. 2001), and so we have a constraint on the time at which the family was evolving towards its current state. As shown in Bottke et al. (2001), the family evolution is dominated by Yarkovsky and YORP effects, as well as by collisions and spin-orbital resonances. The knowledge of the age should constrain some free parameters in various evolutionary models.

The spin-vector properties in an asteroid family were first studied by Slivan (2002) and Slivan et al. (2003), who reveal an anisotropy of spin vectors for ten members of the Koronis family. This was an unexpected result because collisionallyborn population should have an isotropic spin-vector distribution. The peculiar spin-vector alignment in the Koronis family was explained by Vokrouhlický et al. (2003) as a result of the YORP torques and spin-orbital resonances that modified the spin states over the timespan of 2-3 Gyr. The secular $s_{6}$ spin-orbital resonance with Saturn may affect the Koronis family members, according to the

\footnotetext{
2 A thermal recoil force affecting rotating asteroids.
}

numerical simulations, it can (i) capture some objects and create a population of prograde rotators with periods $P \in(4,7) \mathrm{h}$, similar obliquities $\left(42^{\circ}\right.$ to $\left.51^{\circ}\right)$ and also with similar ecliptic longitudes in the ranges of $\left(24^{\circ}\right.$ to $\left.73^{\circ}\right)$ and $\left(204^{\circ}\right.$ to $\left.259^{\circ}\right)$; or (ii) create a group of low-obliquity retrograde rotators with rotational periods $P<5 \mathrm{~h}$ or $P>13 \mathrm{~h}$. The prograde rotators trapped in the $s_{6}$ spin-orbital resonance were referred to by Vokrouhlický et al. (2003) as being in Slivan states. Most members of the Koronis family with known rotational states (determined by the lightcurve inversion by Slivan et al. 2003, 2009; Hanuš et al. 2011, 2013) had the expected properties except for the periods of observed prograde rotators were shifted to higher values of 7-10 h. Rotational states of asteroids that did not match the properties of the two groups were probably reorientated by recent collisions, which are statistically plausible during the family existence for at least a few Koronis members (e.g., asteroid (832) Karin was affected by a collision when a small and young collisional family within the Koronis family was born, Slivan \& Molnar 2012).

Another study of rotational states in an asteroid family was made by Kryszczyńska (2013), who focuses on the Flora family. She distinguishes prograde and retrograde groups of asteroids and reports an excess of prograde rotators. This splitting into two groups is most likely caused by the Yarkovsky effect, while the prograde excess by the secular $v_{6}$ resonance that significantly depopulates the retrograde part of the family. See Fig. 1b, only retrograde rotators can drift via the Yarkovsky/YORP effects towards the resonance.

Future studies of rotational properties of collisional families should reveal the influence of the Yarkovsky and YORP effects, and possibly a capture of asteroids in spin-orbital resonances similar to the case of the Koronis family. The Yarkovsky effect should be responsible for spreading the family in a semi-major axis (retrograde rotators drift from their original positions towards the Sun, on the other hand, prograde rotators drift away from the Sun, i.e. towards larger $a_{\mathrm{p}}$ 's), and the YORP effect should eliminate the spin vectors close to the ecliptic plane.

Disk-integrated photometric observations of asteroids contain information about an object's physical parameters, such as the shape, the sidereal rotational period, and the orientation of the spin axis. Photometry acquired at different viewing geometries and apparitions can be used in many cases in a lightcurve inversion method (e.g., Kaasalainen \& Torppa 2001; Kaasalainen et al. 2001) and a convex 3D shape model including its rotational 
state can be derived. This inverse method uses all available photometric data, both the classical dense-in-time lightcurves or the sparse-in-time data from astrometric surveys. Most of the asteroid models derived by this technique are publicly available in the Database of Asteroid Models from Inversion Techniques (DAMIT $^{3}$, Durech et al. 2010). In February 2013, models of 347 asteroids were included there. About a third of them can be identified as members of various asteroid families. This large number of models of asteroids that belong to asteroid families allows us to investigate the spin-vector properties in at least several families with the largest amount of identified members. Comparison between the observed and synthetic (according to a combined orbital- and spin-evolution model) spin-vector properties could even lead to independent family age estimates.

The paper is organized as follows. In Sect. 2, we investigate the family membership of all asteroids for which we have their models derived by the lightcurve inversion method and present 31 new asteroid models that belong to ten asteroid families. An analysis of spin states within these asteroid families with at least three identified members with known shape models is presented in Sect. 3.1. A combined spin-orbital model for the long-term evolution of a collisional family is described in Sect. 4, where we also compare the synthetic and observed spin-vector properties and constrain the ages of families Flora and Koronis.

\section{Determination of family members}

\subsection{Methods for family membership determination}

For a preliminary family membership determination, we adopted an online catalog published by Nesvorný (2012), who used the hierarchical clustering method ${ }^{4}$ (HCM, Zappalà et al. 1990, 1994). Nesvorný (2012) used two different types of proper elements for the family membership identification: semi-analytic and synthetic. The more reliable dataset is the one derived from synthetic proper elements, which were computed numerically using a more complete dynamical model. The majority of asteroids are present in both datasets. A few asteroids that are only in one of the datasets are included in the study as well (e.g., asteroids (390) Alma in the Eunomia family or (19848) Yeungchuchiu in the Eos family), because at this stage it is not necessary to remove objects that still could be real family members.

The HCM selects a group of objects that are separated in the proper element space by less than a selected distance. However, not all of these objects are actually real members of the collisionallyborn asteroid family. A fraction of objects have orbital elements similar to typical elements of the asteroid family members only by a coincidence, the so-called interlopers. Interlopers can be identified (and removed), for example, by

- inspection of reflectance spectra. Because they are usually of different taxonomic types those that of the family members, we use the SMASSII (Bus \& Binzel 2002) or Tholen taxonomy (Tholen 1984, 1989);

- inspection of colors based on the Sloan Digital Sky Survey Moving Object Catalog 4 (SDSS MOC4, Parker et al. 2008).

\footnotetext{
3 http://astro.troja.mff.cuni.cz/projects/asteroids3D

4 In this method, mutual distances in proper semi-major axis $\left(a_{\mathrm{d}}\right)$, proper eccentricity $\left(e_{\mathrm{d}}\right)$, and proper inclination $\left(i_{\mathrm{d}}\right)$ space are computed. The members of the family are then separated in the proper element space by less than a selected distance (usually, it has a unit of velocity), a free parameter often denoted as "cutoff velocity".
}
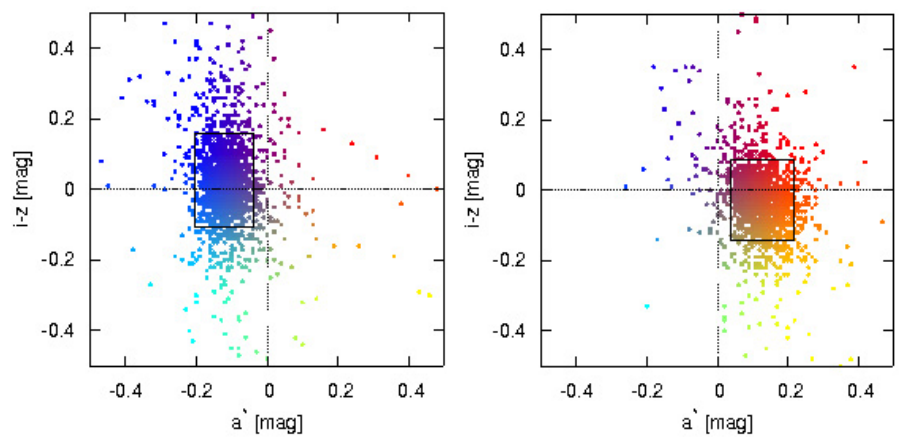

Fig. 2. Dependence of the color indexes $a^{\star}$ and $i-z$ (from the Sloan Digital Sky Survey Moving Object Catalog 4) for a C-type family Themis and S-type family Eunomia. The family corresponds to a compact structure in this parameter space marked by a rectangle. There is a qualitative difference between C- and S-types asteroids.

We used the color indexes $a^{\star}$ and $i-z$, which usually define the core of the family well (see examples for Themis and Eunomia families in Fig. 2), and for each asteroid with available color indexes, we compared values $a^{\star}$ and $i-z$ to those that define the family;

- inspection of albedos based on the WISE data (Masiero et al. 2011);

- construction of a diagram of the proper semi-major axis vs. the absolute magnitude (see Fig. 1), estimating the $V$-shape defined by the Yarkovsky semi-major axis drift and excluding outliers, i.e. relatively large asteroids outside the V-shape (see Vokrouhlický et al. 2006b, for the case of the Eos family). We refer here to the $\left(a_{\mathrm{p}}, H\right)$ border of the family as the border of the $\mathrm{V}$-shape; or by

- construction of a size-frequency distribution (SFD) of the cluster. Some asteroids can be too large to be created within the family and thus are believed to be interlopers (see, e.g., numerical simulations by Michel et al. 2011, who excluded the asteroid (490) Veritas from the Veritas family).

These methods for determining family membership have one common characteristic - we have to determine or choose a range for a quantity that defines the family members (range of spectra, sizes, or distance from the family center), which affects the number of objects we include in the family. Our criteria correspond to the fact that usually $99 \%$ of the objects are within the ranges.

\subsection{New asteroid models}

From the DAMIT database, we adopt 96 models of asteroids that are, according to the HCM, members of collisional families. Currently, we have about 100 new asteroid models that have not yet been published. Here, we present new physical models of 31 asteroids from this sample that are identified as members of asteroid families by the HCM. We choose only asteroids that belong to ten specific families for which we expect a reasonable amount of members, i.e., at least three. These convex shape models are derived by the lightcurve inversion method from combined dense and sparse photometry. The derivation process is similar to the one used in Hanuš et al. (2013). The dense photometry was from two main sources: (i) the Uppsala Asteroid Photometric Catalogue (UAPC ${ }^{5}$, Lagerkvist et al. 1987; Piironen et al. 2001), where lightcurves for about 1000 asteroids are stored; and (ii) the data from a group of individual observers provided by the Minor Planet Center in the

5 http://asteroid.astro.helsinki.fi/ 
A\&A 559, A134 (2013)

Table 1. List of new asteroid models derived from combined dense and sparse data or from sparse data alone.

\begin{tabular}{|c|c|c|c|c|c|c|c|c|c|c|c|}
\hline & Asteroid & $\begin{array}{c}\lambda_{1} \\
\text { [deg] }\end{array}$ & $\begin{array}{c}\beta_{1} \\
\text { [deg] }\end{array}$ & $\begin{array}{c}\lambda_{2} \\
{[\mathrm{deg}]}\end{array}$ & $\begin{array}{c}\beta_{2} \\
\text { [deg] }\end{array}$ & $\begin{array}{c}P \\
\text { [hours] }\end{array}$ & $N_{\text {lc }}$ & $N_{\text {app }}$ & $N_{689}$ & $N_{703}$ & $N_{950}$ \\
\hline 243 & Ida & 259 & -66 & 74 & -61 & 4.633632 & 53 & 6 & 134 & 122 & 25 \\
\hline 364 & Isara & 282 & 44 & 86 & 42 & 9.15751 & 4 & 1 & 98 & 104 & \\
\hline 540 & Rosamunde & 301 & 81 & 127 & 62 & 9.34779 & 3 & 1 & 135 & 83 & \\
\hline 550 & Senta & 63 & -40 & 258 & -58 & 20.5726 & 9 & 1 & 151 & 85 & \\
\hline 553 & Kundry & 197 & 73 & 359 & 64 & 12.6025 & 5 & 1 & 61 & 80 & \\
\hline 621 & Werdandi & 247 & -86 & 66 & -77 & 11.77456 & 12 & 2 & 146 & 71 & \\
\hline 936 & Kunigunde & 47 & 57 & 234 & 50 & 8.82653 & & & 154 & 88 & \\
\hline 951 & Gaspra & 20 & 23 & 198 & 15 & 7.042027 & 71 & 4 & 117 & 89 & \\
\hline 1286 & Banachiewicza & 214 & 62 & 64 & 60 & 8.63043 & & & 81 & 51 & \\
\hline 1353 & Maartje & 266 & 73 & 92 & 57 & 22.9926 & & & 154 & 139 & \\
\hline 1378 & Leonce & 210 & -67 & 46 & -77 & 4.32527 & & & 89 & 113 & \\
\hline 1423 & Jose & 78 & -82 & & & 12.3127 & & & 121 & 134 & \\
\hline 1446 & Sillanpaa & 129 & 76 & 288 & 63 & 9.65855 & 2 & 1 & 76 & 73 & \\
\hline 1464 & Armisticia & 194 & -54 & 35 & -69 & 7.46699 & 2 & 1 & 231 & 67 & \\
\hline 1503 & Kuopio & 170 & -86 & 27 & -61 & 9.9586 & & & 116 & 68 & \\
\hline 1527 & Malmquista & 274 & 80 & & & 14.0591 & & & 49 & 107 & \\
\hline 1618 & Dawn & 39 & -60 & 215 & -51 & 43.219 & & & 93 & 91 & \\
\hline 1633 & Chimay & 322 & 77 & 116 & 81 & 6.59064 & 2 & 1 & 127 & 83 & \\
\hline 1691 & Oort & 45 & 68 & 223 & 58 & 10.2684 & & & 86 & 60 & \\
\hline 1703 & Barry & 46 & -76 & 221 & -71 & 107.04 & & & 89 & 138 & \\
\hline 1805 & Dirikis & 364 & 48 & 188 & 61 & 23.4543 & & & 117 & 91 & \\
\hline 1835 & Gajdariya & 34 & 74 & 204 & 69 & 6.33768 & & & 66 & 86 & \\
\hline 1987 & Kaplan & 357 & -58 & & & 9.45950 & 8 & 2 & 81 & 28 & \\
\hline 2430 & Bruce Helin & 177 & -68 & & & 129.75 & 15 & 1 & & 112 & \\
\hline 3279 & Solon & 268 & -70 & & & 8.1043 & 3 & 1 & & 137 & \\
\hline 3492 & Petra-Pepi & 9 & -57 & 202 & -16 & 46.570 & 15 & 1 & 25 & 111 & \\
\hline 4399 & Ashizuri & 266 & -48 & 45 & -61 & 2.830302 & 4 & 1 & 20 & 84 & \\
\hline 4606 & Saheki & 44 & 59 & 222 & 68 & 4.97347 & 6 & 1 & & 123 & \\
\hline 6159 & $1991 \mathrm{YH}$ & 266 & 67 & 62 & 67 & 10.6590 & 3 & 1 & & 102 & \\
\hline 6262 & Javid & 93 & 76 & 275 & 69 & 8.02054 & 3 & 1 & & 106 & \\
\hline 6403 & Steverin & 246 & 77 & 109 & 73 & 3.49119 & 2 & 1 & & 74 & \\
\hline 7043 & Godart & 73 & 62 & 235 & 80 & 8.4518 & 4 & 1 & & 121 & \\
\hline 7169 & Linda & 11 & -60 & 198 & -61 & 27.864 & 5 & 1 & & 95 & \\
\hline
\end{tabular}

Notes. For each asteroid, the table gives the ecliptic coordinates $\lambda_{1}$ and $\beta_{1}$ of the pole solution with the lowest $\chi^{2}$, the corresponding mirror solution $\lambda_{2}$ and $\beta_{2}$, the sidereal rotational period $P$, the number of dense lightcurves $N_{\text {lc }}$ observed during $N_{\text {app }}$ apparitions, and the number of sparse data points for the corresponding observatory: $N_{689}, N_{703}$, and $N_{950}$. The uncertainty of the sidereal rotational period corresponds to the last decimal place of $P$ and of the pole direction to $5-10^{\circ}$ if we have multi-apparition dense data or $10-20^{\circ}$ if the model is based mainly on sparse data (i.e., only a few dense lightcurves from 1-2 apparitions).

Asteroid Lightcurve Data Exchange Format (ALCDEF ${ }^{6}$, Warner et al. 2009). The sparse-in-time photometry is downloaded from the AstDyS site (Asteroids - Dynamic Site ${ }^{7}$ ). We use data from the three most accurate observatories: USNO-Flagstaff station (IAU code 689), Roque de los Muchachos Observatory, La Palma (IAU code 950), and Catalina Sky Survey Observatory (CSS for short, IAU code 703, Larson et al. 2003).

To increase the number of asteroid models for our study of asteroid families, we performed additional analysis of our previous results of the lightcurve inversion. For many asteroids, we are able to determine a unique rotational period, but get multiple pole solutions (typically 3-5) with similar ecliptic latitudes $\beta$, which is an important parameter. In Hanuš et al. (2011), we presented a reliability test where we checked the physicality of derived solutions by the lightcurve inversion (i.e., if the shape model rotated around its axis with a maximum momentum of inertia). By computing models for all possible pole solutions and by checking their physicality, we removed the pole ambiguity for several asteroids, and thus determined their unique solutions

\footnotetext{
6 http://www.minorplanet.info/alcdef.html

7 http://hamilton.dm.unipi.it/
}

(listed in Table 1). For other asteroids, the pole ambiguity remain and the models give us accurate period values and also rough estimates of ecliptic latitudes $\beta$ (if the biggest difference in latitudes of the models is $<50^{\circ}$ ). We call these models partial and present them in Table 2. For the ecliptic latitude $\beta$, we use the mean value of all different models. We define parameter $\Delta \equiv\left|\beta_{\max }-\beta_{\min }\right| / 2$ as being the estimated uncertainty of $\beta$, where $\beta_{\max }$ and $\beta_{\min }$ are the extremal values within all $\beta$. The threshold for partial models is $\Delta<25^{\circ}$.

We present 31 new models and 24 partial models. References to the dense lightcurves used for the model determination are listed in Table 3. In Sect. 4, we compare the numbers of asteroids in four quadrants of the $\left(a_{\mathrm{p}}, \beta\right)$ diagram (defined by the center of the family and the value $\beta=0^{\circ}$ ) with the same quantities based on the synthetic family population. The uncertainties in $\beta$ are rarely greater than $20^{\circ}$, and the assignment to a specific quadrant is usually not questionable (only in 4 cases out of 136 does the uncertainty interval lie in both quadrants, and most of the asteroids have latitudes $|\beta| \gtrsim 30^{\circ}$ ), and thus give us useful information about the rotational properties in asteroid families. Partial models represent about $20 \%$ of our sample of asteroid models. 
Table 2. List of partial models derived from combined data sets.

\begin{tabular}{rlccccccc}
\hline \hline & Asteroid & $\begin{array}{c}\beta \\
{[\mathrm{deg}]}\end{array}$ & $\begin{array}{c}\Delta \\
{[\mathrm{deg}]}\end{array}$ & $\begin{array}{c}P \\
{[\text { hours }]}\end{array}$ & $N_{\text {lc }}$ & $N_{\text {app }}$ & $N_{689}$ & $N_{703}$ \\
\hline 391 & Ingeborg & -60 & 7 & 26.4145 & 24 & 2 & 141 & 96 \\
502 & Sigune & -44 & 3 & 10.92667 & 9 & 2 & 157 & 52 \\
616 & Elly & 67 & 23 & 5.29771 & 4 & 1 & 101 & 133 \\
1003 & Lilofee & 65 & 10 & 8.24991 & & & 107 & 83 \\
1160 & Illyria & 47 & 23 & 4.10295 & & & 96 & 100 \\
1192 & Prisma & -65 & 14 & 6.55836 & 5 & 1 & 44 & 43 \\
1276 & Ucclia & -49 & 22 & 4.90748 & & & 114 & 45 \\
1307 & Cimmeria & 63 & 9 & 2.820723 & 2 & 1 & 91 & 54 \\
1339 & Desagneauxa & 65 & 17 & 9.37510 & & & 78 & 120 \\
1396 & Outeniqua & 62 & 7 & 3.08175 & 2 & 1 & 112 & 68 \\
1493 & Sigrid & 78 & 7 & 43.179 & & & 78 & 103 \\
1619 & Ueta & 39 & 6 & 2.717943 & 5 & 1 & 122 & 51 \\
1623 & Vivian & -75 & 8 & 20.5235 & & & 77 & 58 \\
1738 & Oosterhoff & -72 & 8 & 4.44896 & & & 109 & 105 \\
1838 & Ursa & 47 & 17 & 16.1635 & & & 102 & 91 \\
2086 & Newell & -60 & 12 & 78.09 & 10 & 1 & 24 & 84 \\
3017 & Petrovic & -73 & 8 & 4.08037 & 3 & 1 & & 114 \\
3786 & Yamada & 56 & 2 & 4.03294 & 3 & 1 & & 71 \\
3896 & Pordenone & -32 & 9 & 4.00366 & 3 & 1 & 22 & 71 \\
4209 & Briggs & -56 & 25 & 12.2530 & 2 & 1 & & 64 \\
4467 & Kaidanovskij & 54 & 13 & 19.1454 & & & 20 & 107 \\
6179 & Brett & -42 & 20 & 9.4063 & 6 & 1 & & 93 \\
7055 & 1989 KB & -61 & 11 & 4.16878 & 7 & 1 & & 117 \\
7360 & Moberg & -18 & 18 & 4.58533 & 3 & 1 & & 103 \\
\hline
\end{tabular}

Notes. For each asteroid, there is the mean ecliptic latitude $\beta$ of the pole direction and its dispersion $\Delta$. The other parameters have the same meaning as in Table 1 . The uncertainty of the sidereal rotational period corresponds to the last decimal place of $P$.

The typical error for the orientation of the pole is (5$\left.10^{\circ}\right) / \cos \beta$ in longitude $\lambda$ and $5-20^{\circ}$ in latitude $\beta$. Both uncertainties depend on the amount, timespan, and quality of used photometry. Models based purely on dense photometry are typically derived from a large number $(\sim 30-50)$ of individual dense lightcurves observed during about five to ten apparitions, and thus the uncertainties of parameters of the rotational state correspond to lower values of the aforementioned range. On the other hand, models based on combined sparse-in-time data have larger uncertainties, owing to the poor photometric quality of the sparse data (corresponds to the upper bound of the aforementioned range).

Models of asteroids (281) Lucretia and (1188) Gothlandia published by Hanuš et al. (2013) were recently determined also by Kryszczyńska (2013) from partly different photometric data sets. Parameters of the rotational state for both models agree within their uncertainties.

The spin vector solution of asteroid (951) Gaspra based on Galileo images obtained during the October 1991 flyby was already published by Davies et al. (1994b). Similarly, the solution of a Koronis-family member (243) Ida based on Galileo images and photometric data was previously derived by Davies et al. (1994a) and Binzel et al. (1993). Here we present convex shape models for both these asteroids. Our derived pole orientations agree within only a few degrees with the previously published values (see Table 5), which again demonstrates the reliability of the lightcurve inversion method.

\subsection{Family members and interlopers}

We revise the family membership assignment by the HCM according to the criteria described above for interlopers or borderline cases. Interlopers are asteroids that do not clearly belong to the family; for example, they have different taxonomic types or incompatible albedos or are far from the $\left(a_{\mathrm{p}}, H\right)$ border. On the other hand, borderline cases cannot be directly excluded from the family, since their physical or orbital properties are just not typical in the context of other members (higher/lower albedos, close to the $\left(a_{\mathrm{p}}, H\right)$ border). These asteroids are possible family members, but can just as easily be interlopers. In the penultimate column of Table 5, we show our revised membership classification for each object ( $\mathrm{M}$ is a member, I an interloper, and B a borderline case), the table also gives the rotational state of the asteroid (the ecliptic coordinates of the pole orientation $\lambda$ and $\beta$ and the period $P$ ), the semi-major axis $a$, the diameter $D$, and the albedo $p_{\mathrm{V}}$ from WISE (Masiero et al. 2011), the SMASS II (Bus \& Binzel 2002), and Tholen (Tholen 1984, 1989) taxonomic types, and the reference to the model).

Although we got several members by the HCM for Vesta and Nysa/Polana families, we excluded these two families from further study of spin states. The Vesta family was created by a cratering event, and thus a majority of the fragments are rather small and beyond the capabilities of the model determination. Most of the models we currently have (recognized by the HCM) are not compatible with the SFD of the Vesta family and thus are interlopers. On the other hand, Nysa/Polana family is a complex of two families (of different age and composition), hence should be treated individually. Additionally, we only have five member candidates for the whole complex, so even if we assign them to the subfamilies, the numbers would be too low to make any valid conclusions.

In Table 4, we list asteroids for which the HCM suggested a membership in families Flora, Koronis, Eos, Eunomia, Phocaea and Alauda, but using the additional methods for the family membership determination described above, we identified them as interlopers or borderline cases. In Fig. 3, we show the $\left(a_{\mathrm{p}}, H\right)$ 

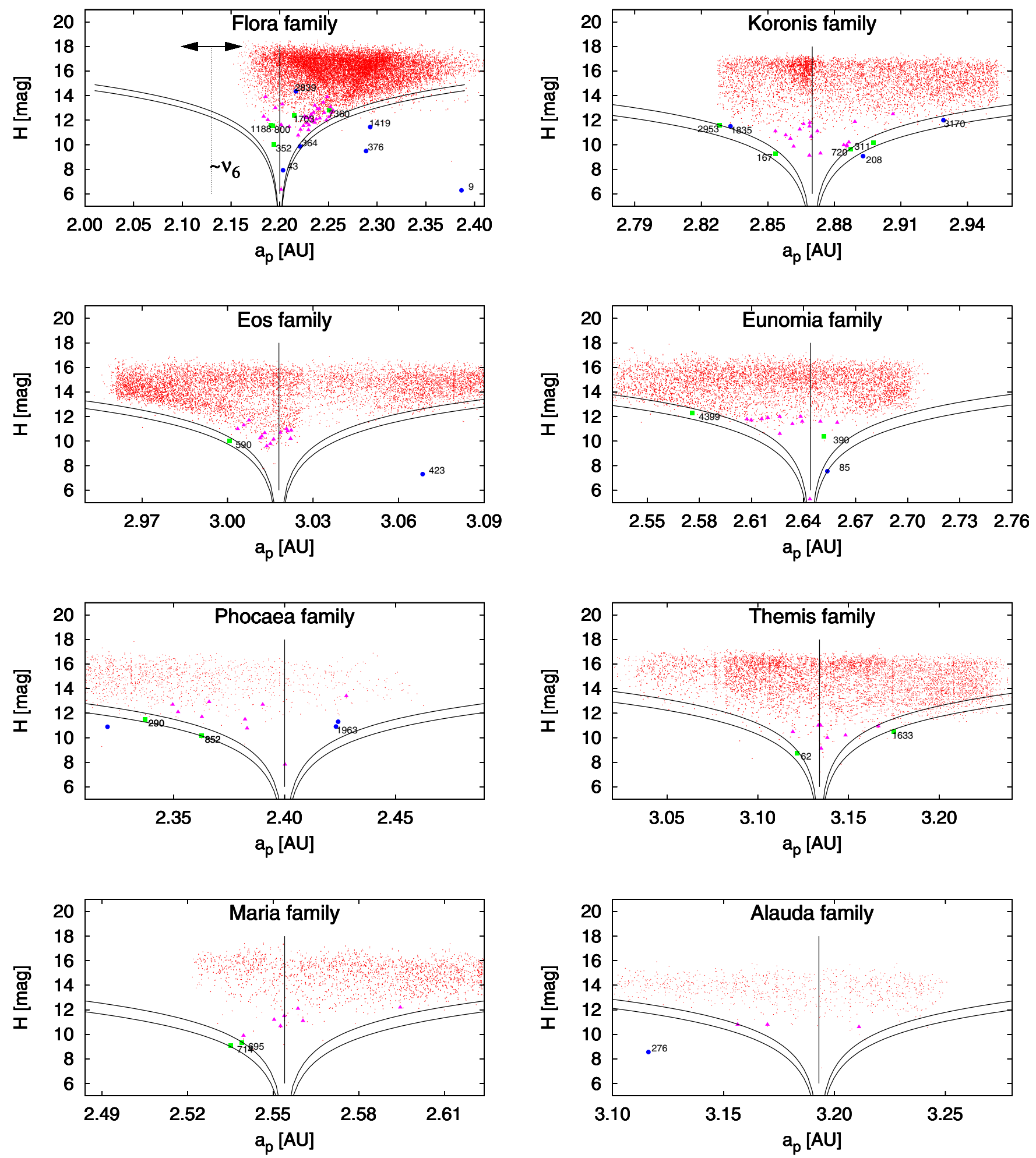

Fig. 3. Dependence of the absolute magnitude $H$ on the proper semi-major axis $a_{\mathrm{p}}$ for the eight families: Flora, Koronis, Eos, Eunomia, Phocaea, Themis, Maria and Alauda with the likely positions of the family centers (vertical lines). We also plot the possible range of the $\left(a_{\mathrm{p}}, H\right)$ borders (two thick lines) of each family for values of the parameter $C$ from Brož et al. (2013) (different values correspond to a different initial extent of the family or different age and magnitude of the Yarkovsky semi-major axis drift.). The pink triangles represent the members from our sample (M), green circles borderline cases (B) and blue circles interlopers (I). Borderline cases and interlopers are identified by several methods including the position in the $\left(a_{\mathrm{p}}, H\right)$ diagram, and thus could also lie close to the center of the family (e.g., in the case of the Flora family).

diagrams for all eight studied families. We plotted the adopted $\left(a_{\mathrm{p}}, H\right)$ border (from Brož et al. 2013) and labeled the members, borderline cases, and interlopers by different colors.
Several asteroids in our sample belong to smaller and younger subclusters within the studied families (e.g., (832) Karin in the Koronis family, (1270) Datura in the Flora 
family, or (2384) Schulhof in the Eunomia family). These subclusters were probably created by secondary collisions. As a result, the spin states of asteroids in these subclusters were randomly reoriented. Because our combined orbital- and spinevolution model (see Sect. 4) includes secondary collisions (reorientations), using asteroids from subclusters in the study of the spin-vector distribution is thus essential: asteroids from subclusters correspond to reoriented asteroids in our synthetic population.

\section{Observed spin vectors in families}

There are eight asteroid families for which we find at least three members (together with borderline cases) in our data set of asteroid models (after the family membership revision, labeled by $\mathrm{M}$ or B in the last column of Table 5) - Flora (38 members), Koronis (23), Eos (16), Eunomia (14), Phocaea (11), Themis (9), Maria (9), and Alauda (3) families. Now that we have the models and membership, we can proceed to the discussion of the spin states in families in general (Sect. 3.1), and for families Flora and Koronis (Sects. 3.2, 3.3).

\subsection{Spin-vector orientations in individual families}

In Fig. 4, we show the dependence of asteroid's pole latitudes in ecliptic coordinates on the semi-major axes. If there are two possible pole solutions for an asteroid, we take the first one in Table 1, because it corresponds to a formally better solution, additionally, latitudes for both ambiguous models are usually similar. To determine the centers of families, we use all members of each family assigned by the HCM, see Figs. 1 and 3. The Eos family has an asymmetric V-shape (the $\left(a_{\mathrm{p}}, H\right)$ diagram), so we compute centers for both wings of the $\mathrm{V}$-shape individually. For the Flora family, we use only the right wing of the V-shape to derive the center, while the left one is strongly affected by the $v_{6}$ secular resonance.

In the study of spin-vector properties in families, we simply use the ecliptic coordinates for the pole orientation: ecliptic longitude $\lambda$ and latitude $\beta$. A formally better approach would be to use the coordinates bound to the orbital plane of the asteroid: orbital longitude $\lambda_{\text {orb }}$ and latitude $\beta_{\text {orb }}$. The orbital latitude can then be easily transformed to obliquity, which directly tells us whether the asteroid rotates in a prograde or retrograde sense. However, for several reasons, we prefer the ecliptic coordinates: (i) most of the asteroids have low inclinations and thus the differences between their ecliptic and orbital latitudes are only a few degrees, and the maximum differences for the families with higher inclination (Eos, Eunomia, Phocaea, Maria) are 20-30 (ii) the orbital coordinates of the pole direction cannot be computed for partial models, because we do not know the ecliptic longitude, these models represent about $20 \%$ of our studied sample; (iii) the positions of the asteroids in the $\left(a_{\mathrm{p}}, \beta\right)$ diagrams (i.e., to which quadrant they belong), namely if they have $\beta>0^{\circ}$ or $\beta<0^{\circ}$, are sufficient information. Because most of the asteroids have latitudes larger than $30^{\circ}$, their positions in the $\left(a_{\mathrm{p}}\right.$, $\beta_{\text {orb }}$ ) are similar (not true only for three asteroids out of 136 ); and (iv) we compare the $\left(a_{\mathrm{p}}, \beta\right)$ diagrams (numbers of objects in the quadrants) between the observed and synthetic populations for ecliptic latitudes, so the consistency is assured.

In general, we observe similar trends for all studied families: (i) larger asteroids are situated in the proximity of the center of the family; (ii) asteroids with $\beta>0^{\circ}$ are usually found to the right of the family center; (iii) asteroids with $\beta<0^{\circ}$ are to the left of the center; (iv) the majority of asteroids have large pole-ecliptic latitudes $\left(|\beta| \gtrsim 30^{\circ}\right)$; and finally (v) some families have a statistically significant excess of asteroids with $\beta>0^{\circ}$ or $\beta<0^{\circ}$.

Case (i) is evident for families Flora, Eunomia, Phocaea, Themis, or Maria. We have no large asteroids in the samples for the remaining families.

Cases (ii) and (iii) are present among all families with the exception of Eos, where all the asteroids are close to the (badly constrained) center. This phenomenon can be easily explained by the Yarkovsky drift, which can change asteroid's semi-major axes $a$; that is, it can increase $a$ of prograde rotators, and decrease $a$ of retrograde once. The magnitude of the Yarkovsky drift is dependent on the asteroid size, is negligible for asteroids with diameters $D \gtrsim 50 \mathrm{~km}$ (the case of Eos), and increases with decreasing diameter. For the Flora, Eunomia, Phocaea, or Maria families, we can see that the smallest asteroids in the sample $(D \sim 5-10 \mathrm{~km})$ can be situated far from the family center, and we can also notice a trend toward decreasing size with increasing distance from the center that probably corresponds to the magnitude of the Yarkovsky effect and the initial velocities $v_{\text {ini }}(D)$ that the objects gained after the break-up.

Observation (iv) is a result of the dynamical evolution of the asteroid's spin vector orientations dominated by the YORP effect, which increases the absolute value of the pole-ecliptic latitude. See papers Hanuš et al. $(2011,2013)$, where this effect is numerically investigated and compared with the observed anisotropic spin vector distribution of the sample of $\sim 300$ MBAs.

Case (v) concerns families Flora, Eunomia, Phocaea, Themis, and Maria. The different number of asteroids with $\beta>0^{\circ}$ and $\beta<0^{\circ}$ among these families is statistically significant and cannot be coincidental. The obvious choice for an explanation are mean-motion or secular resonances. Indeed, the $v_{6}$ secular resonance removed many objects with $\beta>0^{\circ}$ from the Flora family (see Sect. 3.2 for a more thorough discussion). The 8:3 resonance with Jupiter truncated the Eunomia family, which resulted in there being no objects with $a_{\mathrm{p}}>2.70$ AU; similarly, the 3:1 resonance with Jupiter affected the Maria family, for which we do not observe objects with smaller $a_{\mathrm{p}}$ than $2.52 \mathrm{AU}$. The 3:1 resonance with Jupiter is situated near the Phocaea family at $a=2.50 \mathrm{AU}$. Due to the high inclination of objects in the Phocaea family $\left(I \sim 24^{\circ}\right)$, the resonance affects asteroids with $a_{\mathrm{p}}>2.40 \mathrm{AU}$, which corresponds to the probable center of the family. The resonance removed a significant number of objects between 2.40 AU and 2.45 AU, and all objects with larger $a_{\mathrm{p}}$.

The asymmetry of asteroids with $\beta>0^{\circ}$ and $\beta<0^{\circ}$ in the Themis family is caused by a selection effect: in the family, there are no objects with absolute magnitude $H<12$ mag (i.e., large asteroids) and $a_{\mathrm{p}}<3.10 \mathrm{AU}$. On the other hand, with $a_{\mathrm{p}}>3.10 \mathrm{AU}$, there are more than a hundred such asteroids (see Fig. 1a). Our sample of asteroid models derived by the lightcurve inversion method is dominated by larger asteroids, so it is not surprising that we did not derive models for the Themis family asteroids with $a_{\mathrm{p}}<3.10 \mathrm{AU}$. The Flora and Koronis families are also interesting for other aspects, and thus are discussed in more detail in Sects. 3.2 and 3.3.

\subsection{The Flora family}

The Flora cluster is situated in the inner part of the main belt between 2.17-2.40 AU, and its left part (with respect to the $\left(a_{\mathrm{p}}, H\right)$ diagram) is strongly affected by the secular $v_{6}$ resonance with Saturn, which is demonstrated in Fig. 1b. The probable center of the family matches the position of asteroid (8) Flora at $a=2.202$ AU. Because of the relative proximity to the Earth, 

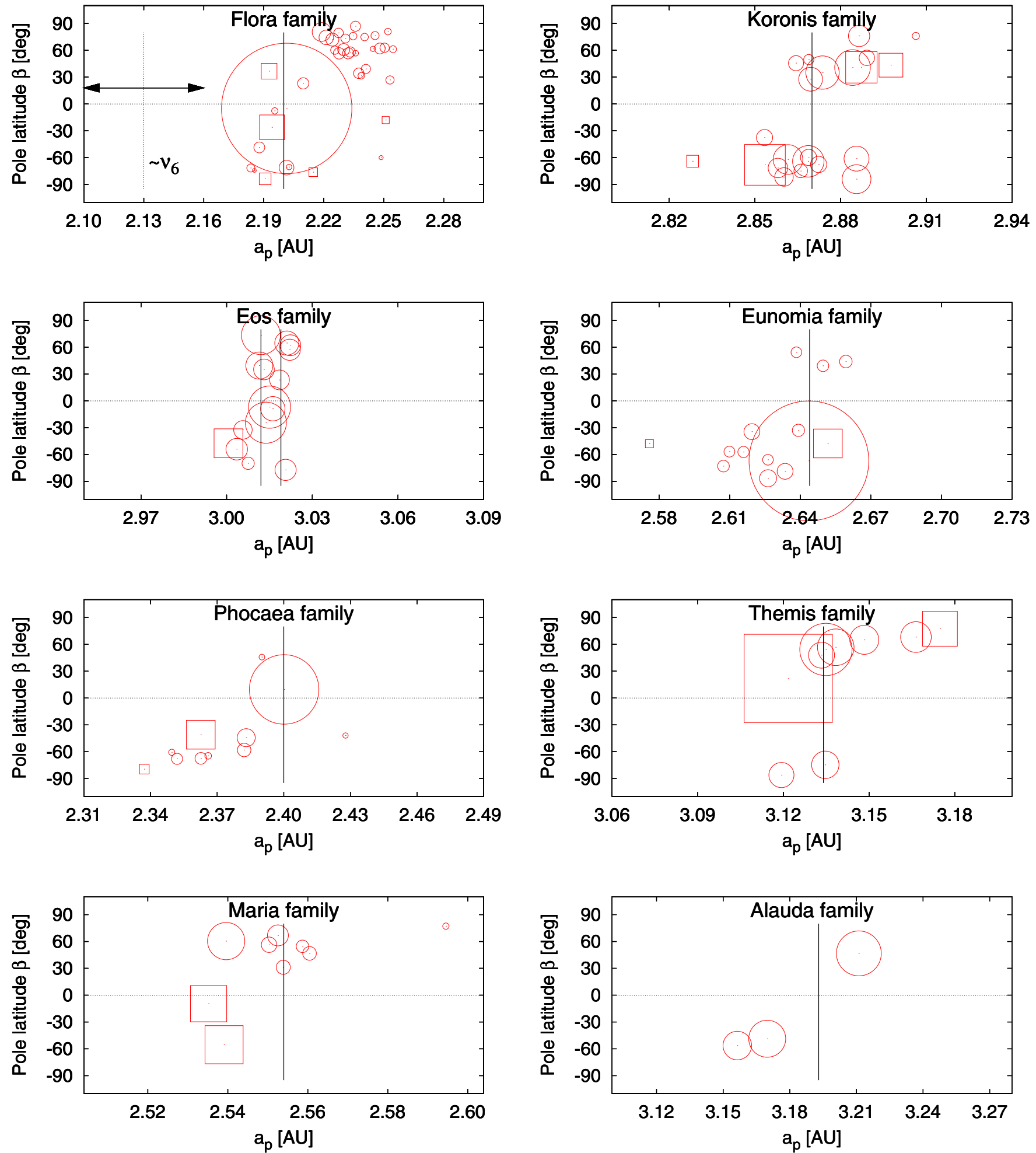

Fig. 4. Dependence of the pole latitude $\beta$ on the proper semi-major axis $a_{\mathrm{p}}$ for eight studied asteroid families: Flora, Koronis, Eos, Eunomia, Phocaea, Themis, Maria, and Alauda. Family members are marked by circles and borderline cases by squares, whose sizes are scaled proportionally to diameters. Only the scale for (15) Eunomia was decreased by half to fit the figure. The vertical lines correspond to the likely centers of the asteroid families, whose uncertainties are usually $<0.01 \mathrm{AU}$. The Eos family has an asymmetric V-shape (the $\left(a_{\mathrm{p}}, H\right)$ border is asymmetric), which makes the center determination harder, so we marked two possible positions. One corresponds to the right $\left(a_{\mathrm{p}}, H\right)$ border, the second to the left border. The uncertainties in $\beta$ are usually $5-20^{\circ}$. In most cases, the value of $|\beta| \gtrsim 30^{\circ}$, hence the quadrant to which the asteroid belongs (defined by the center of the family and the value $\beta=0^{\circ}$ ), is not changed.

more photometric measurements of smaller asteroids are available than for more distant families, and thus more models were derived. So far, we identified 38 models of asteroids that belong to the Flora family (together with borderline cases). 
The majority of asteroids within this family have $\beta>0^{\circ}$ ( $\sim 68 \%$; due to small inclinations of the family members, the majority of the objects with $\beta>0^{\circ}$ are definitely prograde rotators, because their obliquities are between $0^{\circ}$ and $90^{\circ}$ ) and lie to the right of the center of the family, confirming the presence of the Yarkovsky drift. Nine out of twelve asteroids with $\beta<0^{\circ}$ can be found in Fig. 4 near to or to the left of the center of the family. The exceptions are the borderline asteroids (1703) Barry and (7360) Moberg, and asteroid (7169) Linda with $a_{\mathrm{p}}$ close to $2.25 \mathrm{AU}$ (see Fig. 4). The borderline category already suggests that the two asteroids could be possible interlopers, and their rotational state seems to support this statement. However, it is also possible that these asteroids have been reoriented by noncatastrophic collisions. The rotational state of another borderline asteroid (800) Kressmannia also does not agree with the Yarkovsky/YORP predictions, so it could be an interloper (or reoriented). The asteroid (7169) Linda classified as a member could still be an interloper, which was not detected by our methods for interloper removal, or could have recently been reoriented by a noncatastrophic collision. The typical timescale for a reorientation (Farinella et al. 1998, see Eq. (5)) of this 4km-sized asteroid with rotational period $P=27.9 \mathrm{~h}$ is $\tau_{\text {reor }} \sim 500 \mathrm{Myr}$, which is comparable to the age of the family. The depopulation of poles close to the ecliptic plane is also clearly visible.

The $v_{6}$ resonance to the left of the center of the family creates an excess of retrograde rotators not only among the family, but also among the whole main belt population if we use the currently available sample of asteroid models (there are $~ 300$ asteroid models in the DAMIT database). In the Flora family, there are 14 more asteroids with $\beta>0^{\circ}$ than with $\beta<0^{\circ}$ (i.e, we have a prograde excess), which corresponds to about $6 \%$ of the whole sample. This bias needs to be taken into consideration, for example, in the study of rotational properties among MBAs.

The missing asteroids with $\beta<0^{\circ}$ were delivered by this resonance to the orbits crossing the orbits of terrestrial planets and are responsible, for example, for the retrograde excess of the NEAs (La Spina et al. 2004). The $v_{6}$ resonance contributes to the NEA population only by retrograde rotators, and other major mean-motion resonances, such as the 3:1 resonance with Jupiter, deliver both prograde and retrograde rotators in a similar amount.

We did not observe a prograde group of asteroids with similar pole-ecliptic longitudes in the Flora family (i.e., a direct ana$\log$ of the Slivan state in the Koronis family) that was proposed by Kryszczyńska (2013). Although Kryszczyńska (2013) claims that Slivan states are likely to be observed in the Flora family, no corresponding clustering of poles of the prograde rotators is shown, particularly for ecliptic longitudes. We believe that the term Slivan state was used incorrectly there.

\subsection{The Koronis family}

The Koronis family is located in the middle main belt between 2.83-2.95 AU with the center at $a=2.874$ AU. We identified 23 members (together with borderline cases) with determined shape models. The concept given by the Yarkovsky and YORP predictions also work among the Koronis family (asteroids with $\beta<0^{\circ}$ lie to the left from the family center, asteroids with $\beta>0^{\circ}$ to the right, see Fig. 4). In addition to that, Slivan (2002) and Slivan et al. (2003) noticed that prograde rotators have also clustered pole longitudes. These asteroids were trapped in a secular spin-orbital resonance $s_{6}$ and are referred to as being in Slivan states (Vokrouhlický et al. 2003). Several asteroids were later recognized as being incompatible with the Slivan states, such as (832) Karin and (263) Dresda by Slivan \& Molnar (2012). Asteroid (832) Karin is the largest member of a young ( 5.8 Myr, Nesvorný \& Bottke 2004) collisional family that is confined within the larger Koronis family. The spin state of (832) Karin was thus probably affected during this catastrophic event and changed to a random state. Asteroid (263) Dresda could be randomly reoriented by a noncatastrophic collision that is likely to happen for at least a few of 27 asteroids in the Koronis cluster with known spin state solutions, or its initial rotational state and shape did not allow a capture in the resonance. All four borderline asteroids have rotational states that agree with the Yarkovsky/YORP concept, which may support their membership in the Koronis cluster. On the other hand, rotational states of asteroids (277) Elvira and (321) Florentina do not match the expected values, and thus could again be interlopers or be affected by reorientations.

Being trapped in the spin-orbital resonance does not necessarily mean that the asteroid is a member of the Koronis family. It rather indicates that its initial orbital position, the rotational state, and the shape were favorable to being trapped in the resonance. For example, asteroids (311) Claudia, (720) Bohlinia, (1835) Gajdariya, and (3170) Dzhanibekov have expected rotational states but are either rejected from the Koronis family or classified as borderline cases by our membership revision.

\section{Long-term evolution of spin vectors in asteroid families}

Here we present a comparison of the observed spin-vector orientations in several asteroid families with a numerical model of the temporal spin-vector evolutions. We used a combined orbitaland spin-evolution model, which was described in detail in Brož et al. (2011). We need to account for the fact that the Yarkovsky semi-major axis drift is sensitive to the orientation of the spin axis, which is in turn affected by the YORP effect and nondisruptive collisions. This model includes the following processes, which are briefly described in the text: (i) impact disruption; (ii) gravitational perturbations of planets; (iii) the Yarkovsky effect; (iv) the YORP effect; (v) collisions and spin-axis reorientations; and (vi) mass shedding.

Impact disruption. To obtain initial conditions for the family just after the breakup event, we used a very simple model of an isotropic ejection of fragments from the work of Farinella et al. (1994). The distribution of velocities "at infinity" follows the function

$\mathrm{d} N(v) \mathrm{d} v=C^{\prime} v\left(v^{2}+v_{\mathrm{esc}}^{2}\right)^{-(\alpha+1) / 2} \mathrm{~d} v$,

with the exponent $\alpha$ as a free parameter, $C^{\prime}$ a normalization constant and $v_{\text {esc }}$ the escape velocity from the parent body, which is determined by its size $D_{\mathrm{PB}}$ and mean density $\rho_{\mathrm{PB}}$ as $v_{\mathrm{esc}}=$ $\sqrt{(2 / 3) \pi G \rho_{\mathrm{PB}}} D_{\mathrm{PB}}$. The distribution is usually cut at a selected maximum-allowed velocity $v_{\max }$ to prevent outliers. The initial velocities $|v|$ of individual bodies are generated by a straightforward Monte-Carlo code, and the orientations of the velocity vectors $v$ in space are assigned randomly. We also assume that the velocity of fragments is independent of their size.

We must also select initial osculating eccentricity $e_{\mathrm{i}}$ of the parent body, initial inclination $i_{\mathrm{i}}$, as well as true anomaly $f_{\text {imp }}$ and argument of perihelion $\omega_{\text {imp }}$ at the time of impact disruption, which determine the initial shape of the synthetic family just after the disruption of the parent body. 
Gravitational perturbations of planets. Orbital integrations were performed using the SWIFT package (Levison \& Duncan 1994), slightly modified to include necessary online digital filters and a second-order symplectic integrator (Laskar \& Robutel 2001). The second-order symplectic scheme allows us to use a timestep up to $\Delta t=91 \mathrm{~d}$.

Our simulations included perturbations by four outer planets, with their masses, initial positions and velocities taken from the JPL DE405 ephemeris (Standish et al. 1997). We modified the initial conditions of the planets and asteroids by a barycentric correction to partially account for the influence of the terrestrial planets. The absence of the terrestrial planets as perturbers is a reasonable approximation in the middle and outer parts of the main belt (for orbits with $a>2.5 \mathrm{AU}$ and $e<0.6)^{8}$.

Synthetic proper elements are computed as follows. We first apply a Fourier filter to the (nonsingular) orbital elements in a moving window of $0.7 \mathrm{Myr}$ (with steps of $0.1 \mathrm{Myr}$ ) to eliminate all periods smaller than some threshold ( $1.5 \mathrm{kyr}$ in our case). We use a sequence of Kaiser windows as in Quinn et al. (1991).

The filtered signal, which are mean orbital elements, is then passed through a frequency analysis code adapted from Šidlichovský \& Nesvorný (1996) to obtain (planetary) forced and free terms in Fourier representation of the orbital elements. The isolated free terms are what we use as the proper orbital elements.

Yarkovsky effect. Both diurnal and seasonal components of the Yarkovsky accelerations are computed directly in the $N$-body integrator. We used a theory of Vokrouhlický (1998) and Vokrouhlický \& Farinella (1999) for spherical objects (but the magnitude of the acceleration does not differ substantially for nonspherical shapes Vokrouhlický \& Farinella 1998). The implementation within the SWIFT integrator is described in detail by Brož (2006).

YORP effect. The evolution of the orientation of the spin axis and of the angular velocity is given by

$$
\begin{aligned}
\frac{\mathrm{d} \omega}{\mathrm{d} t} & =c f_{i}(\epsilon), \quad i=1 \ldots 200, \\
\frac{\mathrm{d} \epsilon}{\mathrm{d} t} & =c \frac{g_{i}(\epsilon)}{\omega},
\end{aligned}
$$

where $f$ - and $g$-functions describing the YORP effect for a set of 200 shapes were calculated numerically by Čapek \& Vokrouhlický (2004) with the effective radius $R_{0}=1 \mathrm{~km}$ and the bulk density $\rho_{0}=2500 \mathrm{~kg} / \mathrm{m}^{3}$, located on a circular orbit with the semi-major axis $a_{0}=2.5 \mathrm{AU}$. We assigned one of the artificial shapes (denoted by the index $i$ ) to each individual asteroid from our sample. The $f$ - and $g$-functions were then scaled by the factor

$c=c_{\text {YORP }}\left(\frac{a}{a_{0}}\right)^{-2}\left(\frac{R}{R_{0}}\right)^{-2}\left(\frac{\rho_{\text {bulk }}}{\rho_{0}}\right)^{-1}$,

where $a, R$, and $\rho_{\text {bulk }}$ denote the semi-major axis, the radius, and the density of the simulated body, respectively, and $c_{\text {YORP }}$ is a free scaling parameter reflecting our uncertainty in the shape

\footnotetext{
8 For the Flora family located in the inner belt, we should account for terrestrial planets directly, because of mean-motion resonances with Mars, but we decided not do so to speed the computation up. Anyway, the major perturbation we need to account for is the $v_{6}$ secular resonance, which is indeed present in our model.
}

models and the magnitude of the YORP torque, which depends on small-sized surface features (even boulders, Statler 2009) and other simplifications in the modeling of the YORP torque. In Hanuš et al. (2013), we constrained this parameter and find $c_{\text {YORP }}=0.2$ to be the optimal value when comparing the results of the simulation with the observed latitude distribution of main belt asteroids. In our simulation, we used this value for $c_{\text {YORP. }}$

The differential Eqs. (2) and (3) are integrated numerically by a simple Euler integrator. The usual time step is $\Delta t=1000 \mathrm{yr}$.

Collisions and spin-axis reorientations. We neglected the effect of disruptive collisions because we do not want to lose objects during the simulation, but we included spin axis reorientations caused by collisions. We use an estimate of the timescale by Farinella et al. (1998).

$\tau_{\text {reor }}=B\left(\frac{\omega}{\omega_{0}}\right)^{\beta_{1}}\left(\frac{D}{D_{0}}\right)^{\beta_{2}}$,

where $B=84.5 \mathrm{kyr}, \beta_{1}=5 / 6, \beta_{2}=4 / 3, D_{0}=2 \mathrm{~m}$, and $\omega_{0}$ corresponds to period $P=5 \mathrm{~h}$. These values are characteristic of the main belt.

Mass shedding. If the angular velocity approaches a critical value

$\omega_{\text {crit }}=\sqrt{\frac{4}{3} \pi G \rho_{\text {bulk }}}$

we assume a mass shedding event, so we keep the orientation of the spin axis and the sense of rotation, but we reset the orbital period $P=2 \pi / \omega$ to a random value from the interval $(2.5,9) \mathrm{h}$. We also change the assigned shape to a different one, since any change in shape may result in a different YORP effect.

Synthetic Flora, Koronis, and Eos families. In Fig. 5 (top panel), we show a long-term evolution of the synthetic Flora family in the proper semi-major axis $a_{\mathrm{p}}$ vs. the pole latitude $\beta$ plane for objects larger and smaller than $30 \mathrm{~km}$. The values of the model parameters are listed in the figure caption. Larger asteroids do not evolve significantly and remain close to their initial positions. On the other hand, smaller asteroids $(D<30 \mathrm{~km})$ are strongly affected by the Yarkovsky and YORP effects: They drift in the semi-major axis, differently for prograde and retrograde rotators, and their pole orientations become mostly perpendicular to their orbits (corresponding to the proximity of the ecliptic plane for small inclinations). After the simulation at $t=1 \mathrm{Gyr}$, we observe a deficiency of asteroids with $\beta>0^{\circ}$ to the left of the family center and a deficiency of asteroids with $\beta<0^{\circ}$ to the right of the family center.

The asymmetry of the synthetic Flora family with respect to its center (Fig. 5) caused by the secular $v_{6}$ resonance is obvious. The own-right hand quadrant $\left(\beta<0^{\circ}, a_{\mathrm{p}}>2.202 \mathrm{AU}\right)$ still contains many objects for $t=1 \mathrm{Gyr}$, because for some of them the evolution in $\beta$ and $a_{\mathrm{p}}$ is rather small, and others were delivered to this quadrant by collisional reorientations.

The appearance of the evolved proper semi-major axis $a_{\mathrm{p}}$ vs. the pole latitude $\beta$ diagrams for Koronis and Eos families are qualitatively similar to the one of the Flora family. Because the asteroid samples for Koronis and Eos families are dominated by intermediate-sized asteroids $(D \sim 20-50 \mathrm{~km})$, the evolution in $a_{\mathrm{p}}$ and $\beta$ is on average slower than in the Flora family. We show the state of the simulation for Koronis family in $4 \mathrm{Gyr}$ and 

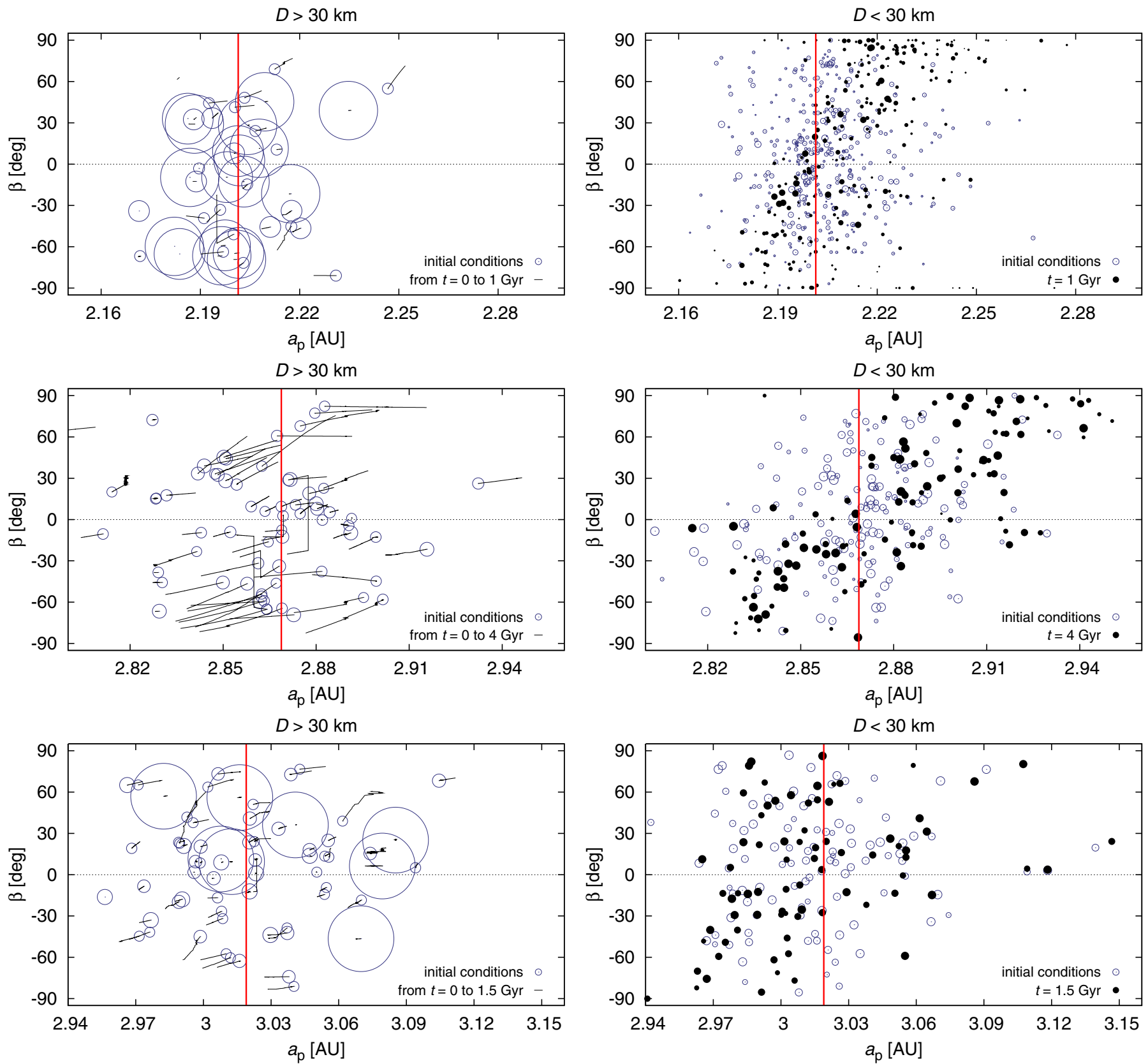

Fig. 5. A simulation of the long-term evolution of the synthetic Flora (top), Koronis (middle) and Eos (bottom) families in the proper semi-major axis $a_{\mathrm{p}}$ vs. the pole latitude $\beta$ plane. Left: objects larger than $D>30 \mathrm{~km}$, which almost do not evolve in $\beta$. Right: objects with $D \leq 30 \mathrm{~km}$, with the initial conditions denoted by empty circles and an evolved state at $1 \mathrm{Gyr}$ denoted by full circles. The sizes of symbols correspond to the actual diameters $D$. The initial conditions for Flora correspond to an isotropic size-independent velocity field with $\alpha=3.25$ and $v_{\text {esc }}=95 \mathrm{~m} \mathrm{~s}^{-1}$, and a uniform distribution of poles (i.e. $\sin \beta$ ). We increase the number of objects 10 times compared to the observed members of the Flora (Koronis and Eos as well) family to improve statistics. We retain their size distribution, of course. The objects in Flora family are discarded from these plots when they left the family region (eccentricity $e_{\mathrm{p}}=0.1$ to 0.18 , inclination $\sin I_{\mathrm{p}}=0.05$ to 0.13 ), because they are affected by strong meanmotion or secular resonances ( $v_{6}$ in this case). Thermal parameters were set as follows: the bulk density $\rho_{\text {bulk }}=2500 \mathrm{~kg} \mathrm{~m}^{-3}$, the surface density $\rho_{\text {surf }}=1500 \mathrm{~kg} \mathrm{~m}^{-3}$, the thermal conductivity $K=0.001 \mathrm{~W} \mathrm{~m}^{-1} \mathrm{~K}^{-1}$, the thermal capacity $C_{\mathrm{t}}=680 \mathrm{~J} \mathrm{~kg}^{-1}$, the Bond albedo $A=0.1$, and the infrared emissivity $\epsilon=0.9$. The time step for the orbital integration is $\mathrm{d} t=91$ days and $\mathrm{d} t_{\text {spin }}=10^{3} \mathrm{yr}$ for the (parallel) spin integration. The parameters for Koronis and Eos are chosen similarly, only for Koronis do we use $v_{\mathrm{esc}}=100 \mathrm{~m} \mathrm{~s}^{-1}$, and $v_{\mathrm{esc}}=225 \mathrm{~m} \mathrm{~s}^{-1}$ and $\rho_{\text {surf }}=2500 \mathrm{~kg} \mathrm{~m}^{-3}$ for Eos.

for Eos in 1.5 Gyr (based on the expected ages). The Eos family thus seems less evolved than the Koronis family.

We also checked the distributions of the proper eccentricities and inclinations of the synthetic Flora/Koronis/Eos objects for whether they (at least roughly) correspond to the observed family. However, the number of objects to compare is fairly low and seems insufficient for any detailed comparison of distributions in 3D space of proper elements $\left(a_{\mathrm{p}}, e_{\mathrm{p}}, \sin I_{\mathrm{p}}\right)$.
Ages of the Flora, Koronis, and Eos families. To quantitatively compare the simulation of the long-term evolution of the synthetic families in the proper semi-major axis $a_{\mathrm{p}}$ vs. the pole latitude $\beta$ plane with the observation, we constructed the following metric: we divide the $\left(a_{\mathrm{p}}, \beta\right)$ plane into four quadrants defined by the center of the family and value $\beta=0^{\circ}$ and compute the ratio $\left(k_{2}+k_{4}\right) /\left(k_{1}+k_{3}\right)$, where $k_{i}$ correspond to the numbers of synthetic objects in quadrants $i(i=1,2,3,4)$. In Fig. 6, we show the 

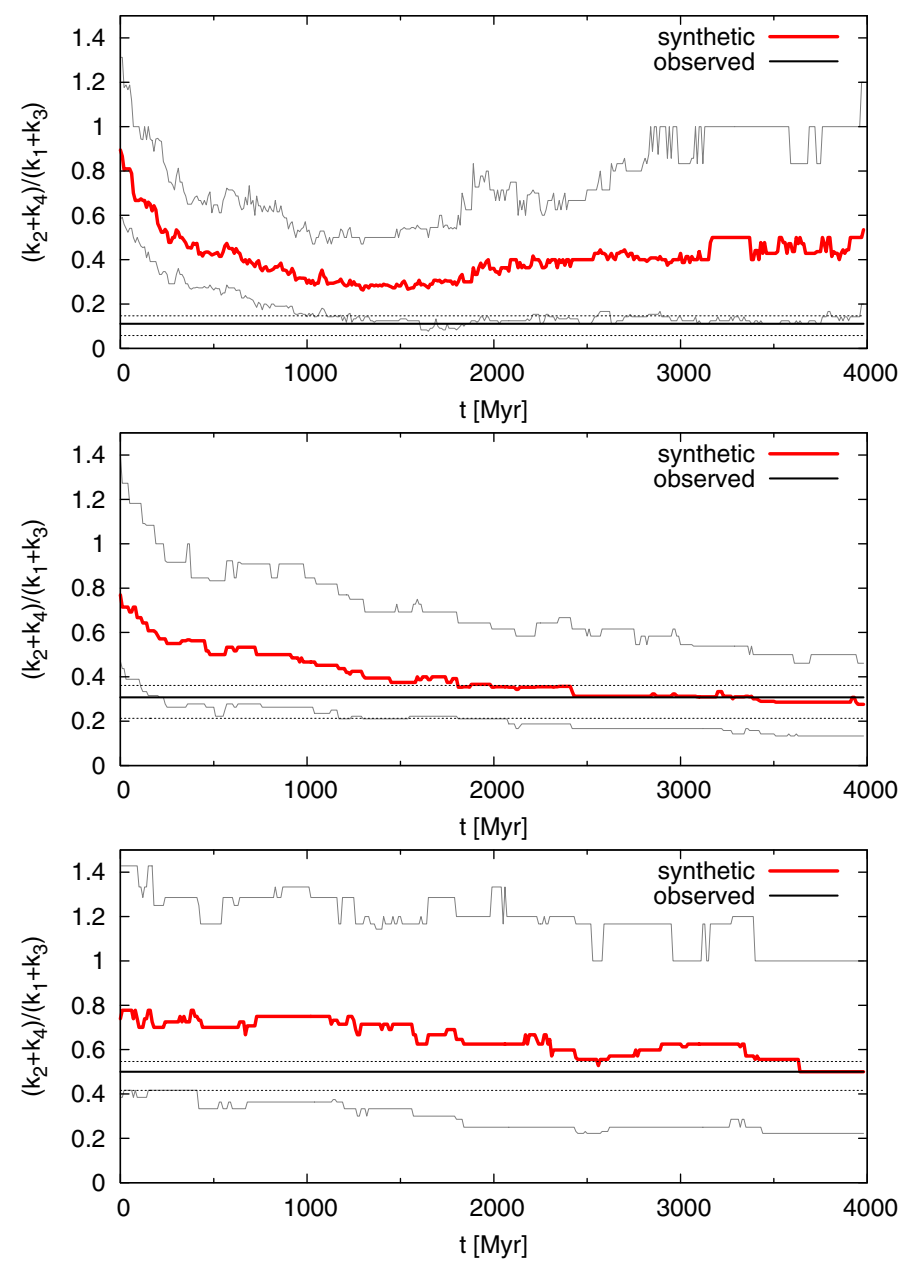

Fig. 6. Time evolution of the metric $\left(k_{2}+k_{4}\right) /\left(k_{1}+k_{3}\right)$, where $k_{i}$ correspond to the numbers of synthetic objects in quadrants $i(i=1,2,3,4)$ that are defined by the center of the family and value $\beta=0^{\circ}$, for synthetic Flora, Koronis, and Eos families (red lines). The spread corresponds to 100 different selections of objects (we simulate 10 times more objects to reach a better statistics). the upper curve denotes the $90 \%$ quantile and the bottom $10 \%$. Thick horizontal line is the observed ratio $\left(k_{2}+k_{4}\right) /\left(k_{1}+k_{3}\right)$ with the uncertainty interval.

evolution of the metric $\left(k_{2}+k_{4}\right) /\left(k_{1}+k_{3}\right)$ during the simulation of families Flora, Koronis, and Eos for all synthetic objects with $D<30 \mathrm{~km}$, and the value of the same metric for the observed population for comparison.

For the Koronis family (middle panel), the synthetic ratio reaches the observed one after $t=2.5 \mathrm{Gyr}$ and remains similar until the end of the simulation at $t=4$ Gyr. Bottke et al. (2001) published the age $t=(2.5 \pm 1.0) \mathrm{Gyr}$ for the Koronis family. Unfortunately, we cannot constrain the age of the Eos family from this simulation owing to objects with the relatively small evolution in $a_{\mathrm{p}}$ and $\beta$. The fit for the Flora family is not ideal, and the reason could be differences in the initial velocity field or the true anomaly $f_{\text {imp }}$ of the impact. The best agreement is for the age $t=(1.0 \pm 0.5) \mathrm{Gyr}$, which is approximately in agreement with the dynamical age in Nesvorný et al. (2005): (1.5 \pm 0.5$)$ Gyr.

\section{Conclusions}

We have identified 152 asteroids for which we have convex shape models, and simultaneously the HCM identifies them as members of ten collisional families. Owing to a large number of expected interlopers in families Vesta and Nysa/Polana, we excluded these families from the study of the rotational properties. In the remaining sample of asteroids from eight families, we identified $\sim 20 \%$ of objects that are interlopers or borderline cases (see Table 4). We used several methods, described in Sect. 2.1, for their identification. The borderline cases are still possible members of the families and thus were included in our study of the spin-vector distribution.

From the dependence of the asteroid's pole latitudes on the semi-major axes, plotted in Fig. 4, we can see fingerprints of families spreading in $a$ and spin axis evolution due to Yarkovsky and YORP effects: Asteroids with $\beta<0^{\circ}$ lie on the left side of the center of the family, and asteroids with $\beta>0^{\circ}$ on the right side. The asymmetry with respect to the family centers is in most cases caused by various resonances that cut the families, and in the case of Themis family, a selection effect is responsible.

However, we did not observe perfect agreement with the Yarkovsky and YORP effects predictions. A few individual objects (eight) that have incompatible rotational states could (i) be incorrectly determined; (ii) be interlopers; (iii) have initial rotational states that only cause a small evolution in the $\left(a_{\mathrm{p}}, \beta\right)$ space (i.e., they are close to their initial positions after the break-up); or (iv) be recently reoriented by collisional events.

In the case of the Flora family, significantly fewer asteroids with $\beta<0^{\circ}(\sim 32 \%)$ than with $\beta>0^{\circ}(\sim 68 \%)$ are present. The secular $v_{6}$ resonance is responsible for this strong deficit, because objects with $\beta<0^{\circ}$ are drifting towards this resonance and are subsequently removed from the family. They become part of the NEAs population where they create an excess of retrograde rotators. We did not find any analog of the Slivan states (observed in the Koronis family) among any other of the studied families.

We simulated a long-term evolution of the synthetic Flora, Koronis, and Eos families (Fig. 5) in the proper semi-major axis $a_{\mathrm{p}}$ vs the pole latitude $\beta$ plane and compared the results with the properties of observed asteroid families. We obtained a good qualitative agreement between the observed and synthetic spinvector distributions. For all three families, we computed evolution of the number of objects in the four quadrants of the families in the $\left(a_{\mathrm{p}}, \beta\right)$ diagram, and we estimated ages for families Flora $(1.0 \pm 0.5) \mathrm{Gyr}$ and Koronis $(2.5$ to $4 \mathrm{Gyr})$ that agree with previously published values. However, we did not estimate the age of the Eos family due to a small evolution of the objects in the $\left(a_{\mathrm{p}}, \beta\right)$ diagram.

The uncertainties seem to be dominated by the observed quadrant ratios. We expect that increasing the sample size by a factor of 10 would decrease the relative uncertainty by a factor of about 3, which is a good motivation for further work on this subject.

Acknowledgements. The work of J.H. and J.D. has been supported by grants GACR P209/10/0537 and P209/12/0229 of the Czech Science Foundation, and the work of J.D. and M.B. by the Research Program MSM0021620860 of the Czech Ministry of Education. The work of M.B. has been also supported by grant GACR 13-013085 of the Czech Science Foundation.

\section{References}

Binzel, R. P., Slivan, S. M., Magnusson, P., et al. 1993, Icarus, 105, 310

Bottke, W. F., Vokrouhlický, D., Brož, M., Nesvorný, D., \& Morbidelli, A. 2001, Science, 294, 1693

Bottke, J. W. F., Vokrouhlický, D., Rubincam, D. P., \& Nesvorný, D. 2006, Ann. Rev. Earth Planet. Sci., 34, 157

Brinsfield, J. W. 2008a, Minor Planet Bulletin, 35, 179

Brinsfield, J. W. 2008b, Minor Planet Bulletin, 35, 119

Brinsfield, J. W. 2009, Minor Planet Bulletin, 36, 127 
Brož, M. 2006, Ph.D. Thesis, Charles University in Prague

Brož, M., Vokrouhlický, D., Morbidelli, A., Nesvorný, D., \& Bottke, W. F. 2011, MNRAS, 414, 2716

Brož, M., Morbidelli, A., Bottke, W. F., et al. 2013, A\&A, 551, A117

Bus, S. J., \& Binzel, R. P. 2002, Icarus, 158, 146

Čapek, D., \& Vokrouhlický, D. 2004, Icarus, 172, 526

Davies, M. E., Colvin, T. R., Belton, M. J. S., et al. 1994a, in AAS/Division for Planetary Sciences Meeting Abstracts \#26, BAAS, 26, 1154

Davies, M. E., Colvin, T. R., Belton, M. J. S., Veverka, J., \& Thomas, P. C. 1994b, Icarus, 107, 18

Drummond, J., Eckart, A., \& Hege, E. K. 1988, Icarus, 73, 1

Durech, J., Scheirich, P., Kaasalainen, M., et al. 2007, in IAU Symp. 236, eds. G. B. Valsecchi, D. Vokrouhlický, \& A. Milani, 191

Ďurech, J., Kaasalainen, M., Warner, B. D., et al. 2009, A\&A, 493, 291

Durech, J., Sidorin, V., \& Kaasalainen, M. 2010, A\&A, 513, A46

Durech, J., Kaasalainen, M., Herald, D., et al. 2011, Icarus, 214, 652

Durkee, R. I. 2010, Minor Planet Bulletin, 37, 125

Farinella, P., Froeschlé, C., \& Gonczi, R. 1994, in Asteroids, Comets, Meteors 1993, eds. A. Milani, M. di Martino, \& A. Cellino, IAU Symp., 160, 205

Farinella, P., Vokrouhlický, D., \& Hartmann, W. K. 1998, Icarus, 132, 378

Giblin, I., Martelli, G., Farinella, P., et al. 1998, Icarus, 134, 77

Hanuš, J., Ďurech, J., Brož, M., et al. 2011, A\&A, 530, A134

Hanuš, J., Durech, J., Brož, M., et al. 2013, A\&A, 551, A67

Higgins, D. 2011, Minor Planet Bulletin, 38, 41

Higgins, D., \& Goncalves, R. M. D. 2007, Minor Planet Bulletin, 34, 16

Johansen, A., \& Lacerda, P. 2010, MNRAS, 404, 475

Kaasalainen, M., \& Torppa, J. 2001, Icarus, 153, 24

Kaasalainen, M., Torppa, J., \& Muinonen, K. 2001, Icarus, 153, 37

Kaasalainen, M., Torppa, J., \& Piironen, J. 2002, Icarus, 159, 369

Koff, R. A., Brincat, S. M., Stephens, R. D., \& Pravec, P. 2001, Minor Planet Bulletin, 28, 46

Kryszczyńska, A. 2013, A\&A, 551, A102

Kryszczyńska, A., La Spina, A., Paolicchi, P., et al. 2007, Icarus, 192, 223

La Spina, A., Paolicchi, P., Kryszczyńska, A., \& Pravec, P. 2004, Nature, 428, 400

Lagerkvist, C., Barucci, M. A., Capria, M. T., et al. 1987, Asteroid photometric catalogue (Roma: CNR)

Larson, S., Beshore, E., Hill, R., et al. 2003, in AAS/Division for Planetary Sciences Meeting Abstracts \#35, BAAS, 35, 982

Laskar, J., \& Robutel, P. 2001, Celest. Mech. Dyn. Astron., 80, 39

Levison, H. F., \& Duncan, M. J. 1994, Icarus, 108, 18

Magnusson, P. 1986, Icarus, 68, 1

Marchis, F., Kaasalainen, M., Hom, E. F. Y., et al. 2006, Icarus, 185, 39

Marciniak, A., Michałowski, T., Kaasalainen, M., et al. 2007, A\&A, 473, 633

Masiero, J. R., Mainzer, A. K., Grav, T., et al. 2011, ApJ, 741, 68

Michel, P., Jutzi, M., Richardson, D. C., \& Benz, W. 2011, Icarus, 211, 535

Nesvorný, D. 2012, in NASA Planetary Data System, 189

Nesvorný, D., \& Bottke, W. F. 2004, Icarus, 170, 324

Nesvorný, D., \& Morbidelli, A. 1998, AJ, 116, 3029

Nesvorný, D., Jedicke, R., Whiteley, R. J., \& Ž. Ivezić. 2005, Icarus, 173, 132

Oey, J. 2006, Minor Planet Bulletin, 33, 96

Paolicchi, P., \& Kryszczyńska, A. 2012, Planet. Space Sci., 73, 70
Parker, A., Ž. Ivezić, Jurić, M., et al. 2008, Icarus, 198, 138

Piironen, J., Lagerkvist, C., Torppa, J., Kaasalainen, M., \& Warner, B. 2001, BAAS, 33, 1562

Polishook, D., Ofek, E. O., Waszczak, A., et al. 2012, MNRAS, 421, 2094

Pravec, P., Harris, A. W., Kušnirák, P., Galád, A., \& Hornoch, K. 2012, Icarus, 221,365

Pravec, P., Harris, A. W., \& Michałowski, T. 2002, Asteroids III, 113

Quinn, T. R., Tremaine, S., \& Duncan, M. 1991, AJ, 101, 2287

Rau, A., Kulkarni, S. R., Law, N. M., et al. 2009, PASP, 121, 1334

Rubincam, D. P. 2000, Icarus, 148, 2

Šidlichovský, M., \& Nesvorný, D. 1996, Celest. Mech. Dyn. Astron., 65, 137

Skoglöv, E., \& Erikson, A. 2002, Icarus, 160, 24

Slivan, S. M. 2002, Nature, 419, 49

Slivan, S. M., \& Molnar, L. A. 2012, Icarus, 220, 1097

Slivan, S. M., Binzel, R. P., Crespo da Silva, L. D., et al. 2003, Icarus, 162, 285

Slivan, S. M., Binzel, R. P., Kaasalainen, M., et al. 2009, Icarus, 200, 514

Standish, E. M., Newhall, X. X., Williams, J. G., \& Folkner, W. M. 1997, JPL Planetary and Lunar Ephemerides, CD-ROM published by Willmann-Bell, Inc., Richmond, Virginia

Statler, T. S. 2009, Icarus, 202, 502

Stephens, R. D. 2003, Minor Planet Bulletin, 30, 1

Stephens, R. D. 2005, Minor Planet Bulletin, 32, 66

Stephens, R. D. 2007a, Minor Planet Bulletin, 34, 31

Stephens, R. D. 2007b, Minor Planet Bulletin, 34, 102

Stephens, R. D. 2007c, Minor Planet Bulletin, 34, 64

Stephens, R. D. 2011a, Minor Planet Bulletin, 38, 211

Stephens, R. D. 2011b, Minor Planet Bulletin, 38, 23

Strabla, L., Quadri, U., Girelli, R., \& Pilcher, F. 2012, Minor Planet Bulletin, 39, 154

Tholen, D. J. 1984, Ph.D. Thesis, Tucson: Arizona Univ.

Tholen, D. J. 1989, in Asteroids II, ed. R. P. Binzel, T. Gehrels, \& M. S. Matthews, 1139

Torppa, J., Kaasalainen, M., Michałowski, T., et al. 2003, Icarus, 164, 346

Vokrouhlický, D. 1998, A\&A, 335, 1093

Vokrouhlický, D., \& Farinella, P. 1998, AJ, 116, 2032

Vokrouhlický, D., \& Farinella, P. 1999, AJ, 118, 3049

Vokrouhlický, D., Nesvorný, D., \& Bottke, W. F. 2003, Nature, 425, 147

Vokrouhlický, D., Brož, M., Michałowski, T., et al. 2006a, Icarus, 180, 217

Vokrouhlický, D., Nesvorný, D., \& Bottke, W. F. 2006b, Icarus, 184, 1

Vokrouhlický, D., Durech, J., Michałowski, T., et al. 2009, A\&A, 507, 495

Warner, B. 2001, Minor Planet Bulletin, 28, 40

Warner, B. D. 2004, Minor Planet Bulletin, 31, 19

Warner, B. D. 2005, Minor Planet Bulletin, 32, 29

Warner, B. D. 2006, Minor Planet Bulletin, 33, 82

Warner, B. D. 2009, Minor Planet Bulletin, 36, 172

Warner, B. D. 2010, Minor Planet Bulletin, 37, 112

Warner, B. D. 2011, Minor Planet Bulletin, 38, 63

Warner, B. D., \& Pray, D. P. 2009, Minor Planet Bulletin, 36, 166

Warner, B. D., Harris, A. W., \& Pravec, P. 2009, Icarus, 202, 134

Zappalà, V., Cellino, A., Farinella, P., \& Knežević, Z. 1990, AJ, 100, 2030

Zappalà, V., Cellino, A., Farinella, P., \& Milani, A. 1994, AJ, 107, 772 
Table 3. Observations not included in the UAPC used for successful model determinations.

\begin{tabular}{|c|c|c|c|c|}
\hline \multicolumn{2}{|r|}{ Asteroid } & \multirow{2}{*}{$\frac{\text { Date }}{20095-200905}$} & \multirow{2}{*}{$\frac{\text { Observer }}{\text { Warner }(2009)}$} & \multirow{2}{*}{$\frac{\text { Observatory (MPC code) }}{\text { Palmer Divide Observatorv (716) }}$} \\
\hline 364 & Isara & & & \\
\hline 391 & Ingeborg & $20008-200012$ & Koff et al. (2001) & Antelope Hills Observatory, Bennett (H09) \\
\hline 502 & Sigune & $20076-20076$ & Stephens (2007b) & Goat Mountain Astronomical Research Station (G79) \\
\hline 553 & Kundry & $200412-20051$ & Stephens (2005) & Goat Mountain Astronomical Research Station (G79) \\
\hline \multirow[t]{2}{*}{616} & Elly & $20101-20101$ & Warner (2010) & Palmer Divide Observatory (716) \\
\hline & & $20102-20102$ & Durkee (2010) & Shed of Science Observatory, USA (H39) \\
\hline \multirow[t]{2}{*}{621} & Werdandi & 2012122.9 & Strabla et al. (2012) & Bassano Bresciano Observatory (565) \\
\hline & & $20121-20122$ & Strabla et al. (2012) & Organ Mesa Observatory (G50) \\
\hline 1307 & Chimmeria & 2004 9-20049 & Warner (2005) & Palmer Divide Observatory (716) \\
\hline 1396 & Outeniqua & $20063-20063$ & Warner (2006) & Palmer Divide Observatory (716) \\
\hline 1446 & Sillanpaa & 2009 3-2009 3 & Higgins ${ }^{1}$ & Hunters Hill Observatory, Ngunnawal (E14) \\
\hline 1464 & Armisticia & $20081-20081$ & Brinsfield (2008b) & Via Capote Sky Observatory, Thousand Oaks (G69) \\
\hline \multirow[t]{2}{*}{1619} & Ueta & $20109-201010$ & Higgins (2011) & Hunters Hill Observatory, Ngunnawal (E14) \\
\hline & & $20109-20109$ & Stephens (2011b) & Goat Mountain Astronomical Research Station (G79) \\
\hline 1633 & Chimay & $20084-20084$ & Brinsfield (2008a) & Via Capote Sky Observatory, Thousand Oaks (G69) \\
\hline \multirow[t]{2}{*}{1987} & Kaplan & $200010-200010$ & Warner $(2001,2011)$ & Palmer Divide Observatory (716) \\
\hline & & $201112-201112$ & Warner & Palmer Divide Observatory (716) \\
\hline 2086 & Newell & 2007 1-2007 2 & Stephens (2007c) & Goat Mountain Astronomical Research Station (G79) \\
\hline 2403 & Bruce Helin & 2006 9-2006 9 & Higgins ${ }^{1}$ & Hunters Hill Observatory, Ngunnawal (E14) \\
\hline 3279 & Solon & $200611-200611$ & Stephens (2007a) & Goat Mountain Astronomical Research Station (G79) \\
\hline 3492 & Petra-Pepi & $20116-20117$ & Stephens (2011a) & Goat Mountain Astronomical Research Station (G79) \\
\hline 3786 & Yamada & $20027-20028$ & Stephens (2003) & Goat Mountain Astronomical Research Station (G79) \\
\hline 3896 & Pordenone & $200710-200710$ & Higgins ${ }^{1}$ & Hunters Hill Observatory, Ngunnawal (E14) \\
\hline 4209 & Briggs & $20039-20039$ & Warner (2004) & Palmer Divide Observatory (716) \\
\hline 4399 & Ashizuri & $20086-20086$ & Brinsfield (2008a) & Via Capote Sky Observatory, Thousand Oaks (G69) \\
\hline 4606 & Saheki & $20091-20093$ & Brinsfield (2009) & Via Capote Sky Observatory, Thousand Oaks (G69) \\
\hline 6159 & $1991 \mathrm{YH}$ & $20063-20063$ & Warner (2006) & Palmer Divide Observatory (716) \\
\hline 6179 & Brett & 2009 4-2009 4 & Warner \& Pray (2009) & Palmer Divide Observatory (716) \\
\hline 6262 & Javid & $20102-20102$ & $\mathrm{PTF}^{2}$ & \\
\hline 6403 & Steverin & 2004 9-20049 & Warner (2005) & Palmer Divide Observatory (716) \\
\hline \multirow[t]{2}{*}{7043} & Godart & $20088-20088$ & Durkee & Shed of Science Observatory, USA (H39) \\
\hline & & $20088-20089$ & Pravec et al. (2012) & Goat Mountain Astronomical Research Station (G79) \\
\hline \multirow[t]{2}{*}{7055} & $1989 \mathrm{~KB}$ & $20075-20075$ & Stephens (2007b) & Goat Mountain Astronomical Research Station (G79) \\
\hline & & $20075-20076$ & Higgins ${ }^{1}$ & Hunters Hill Observatory, Ngunnawal (E14) \\
\hline 7169 & Linda & $20068-20068$ & Higgins \& Goncalves (2007) & Hunters Hill Observatory, Ngunnawal (E14) \\
\hline 7360 & Moberg & $20064-20064$ & Oey (2006) & Leura (E17) \\
\hline
\end{tabular}

Notes. ${ }^{(1)}$ On line at http://www.david-higgins.com/Astronomy/asteroid/lightcurves.htm ${ }^{(2)}$ Palomar Transient Factory survey (Rau et al. 2009), data taken from Polishook et al. (2012). 
J. Hanuš et al.: Spin vectors in asteroid families

Table 4. List of asteroids for which the HCM alone suggests membership in families Flora, Koronis, Eos, Eunomia, Phocaea, and Alauda.

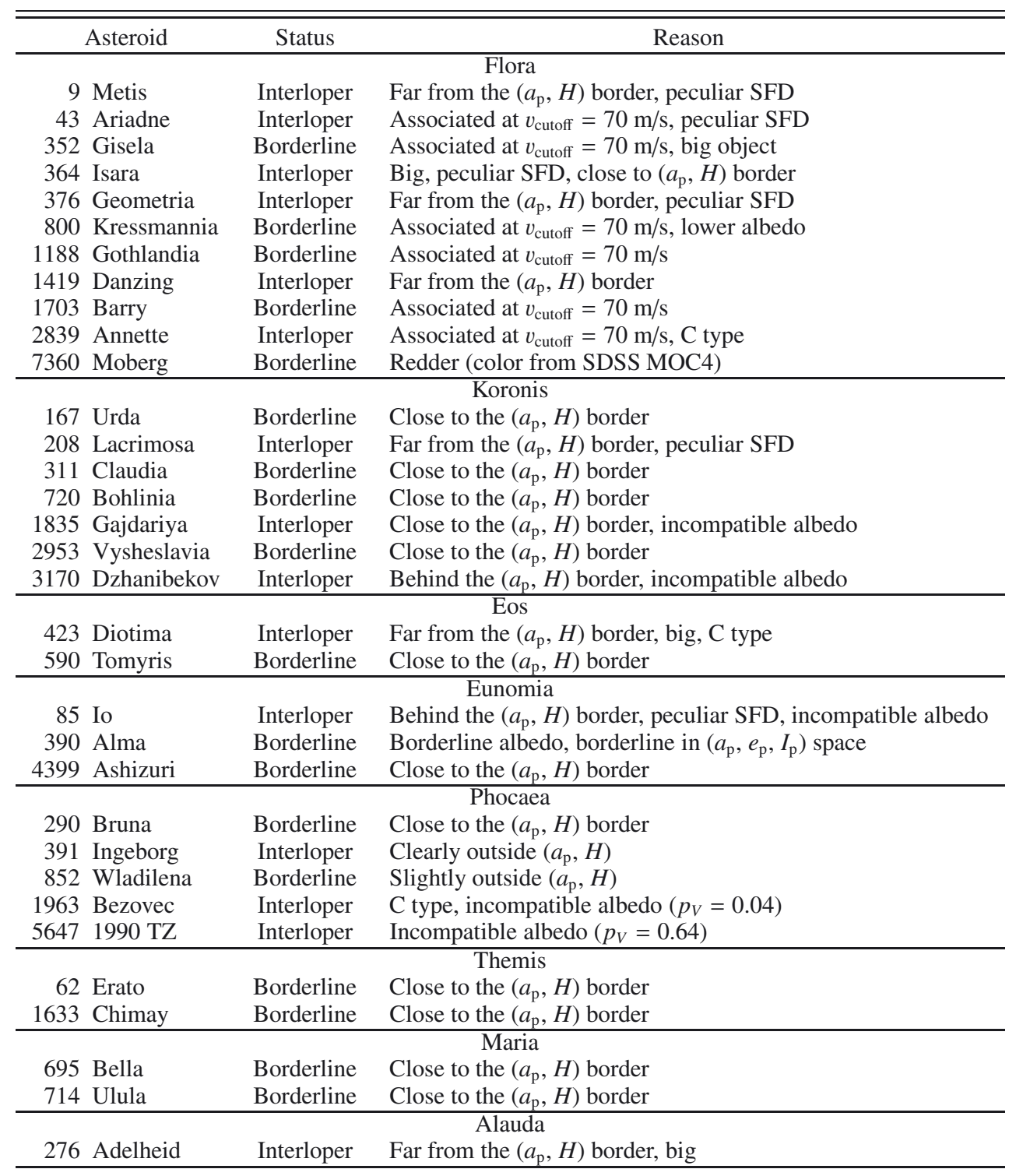

Notes. By additional methods for determining family membership we identify them as interlopers or borderline cases. We also give the name of the asteroid, the family membership according the HCM, if it is an interloper or a borderline case and the reason. Peculiar SFD means a size frequency distribution that is incompatible with the SFD typically created by catastrophic collisions or cratering events (i.e., a large remnant, large fragment, and steep slope). Quantity $v_{\text {cutoff }}$ corresponds to the cutoff value of the HCM for a particular family. 


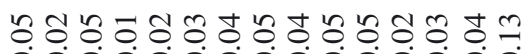

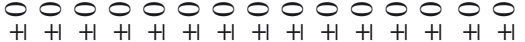

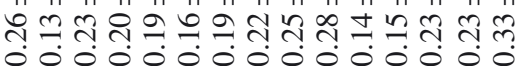

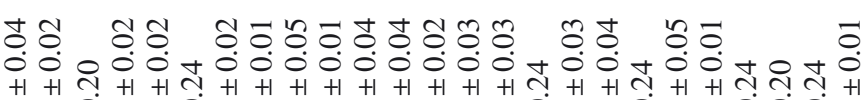

jo

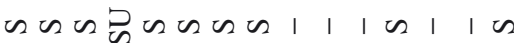
।

n $\infty$

$$
\text { a } 1
$$

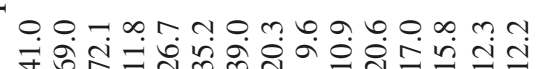

우웅

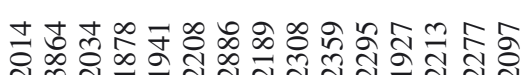

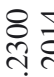

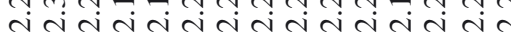

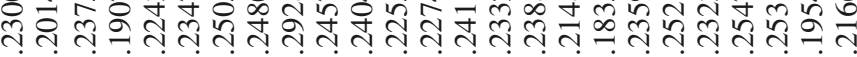

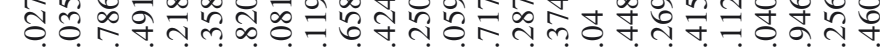

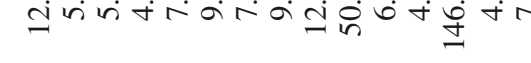

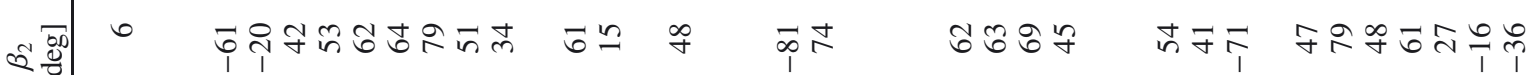

ㅁํㅇ బ

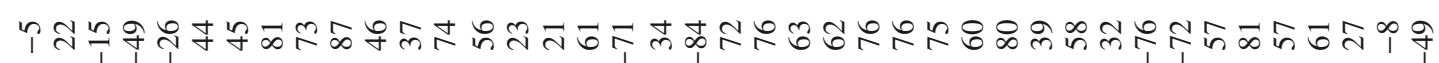

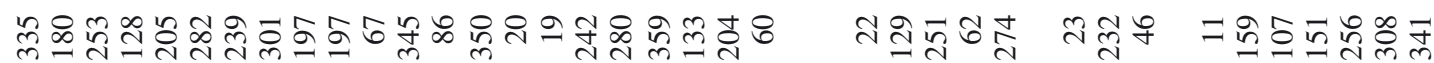




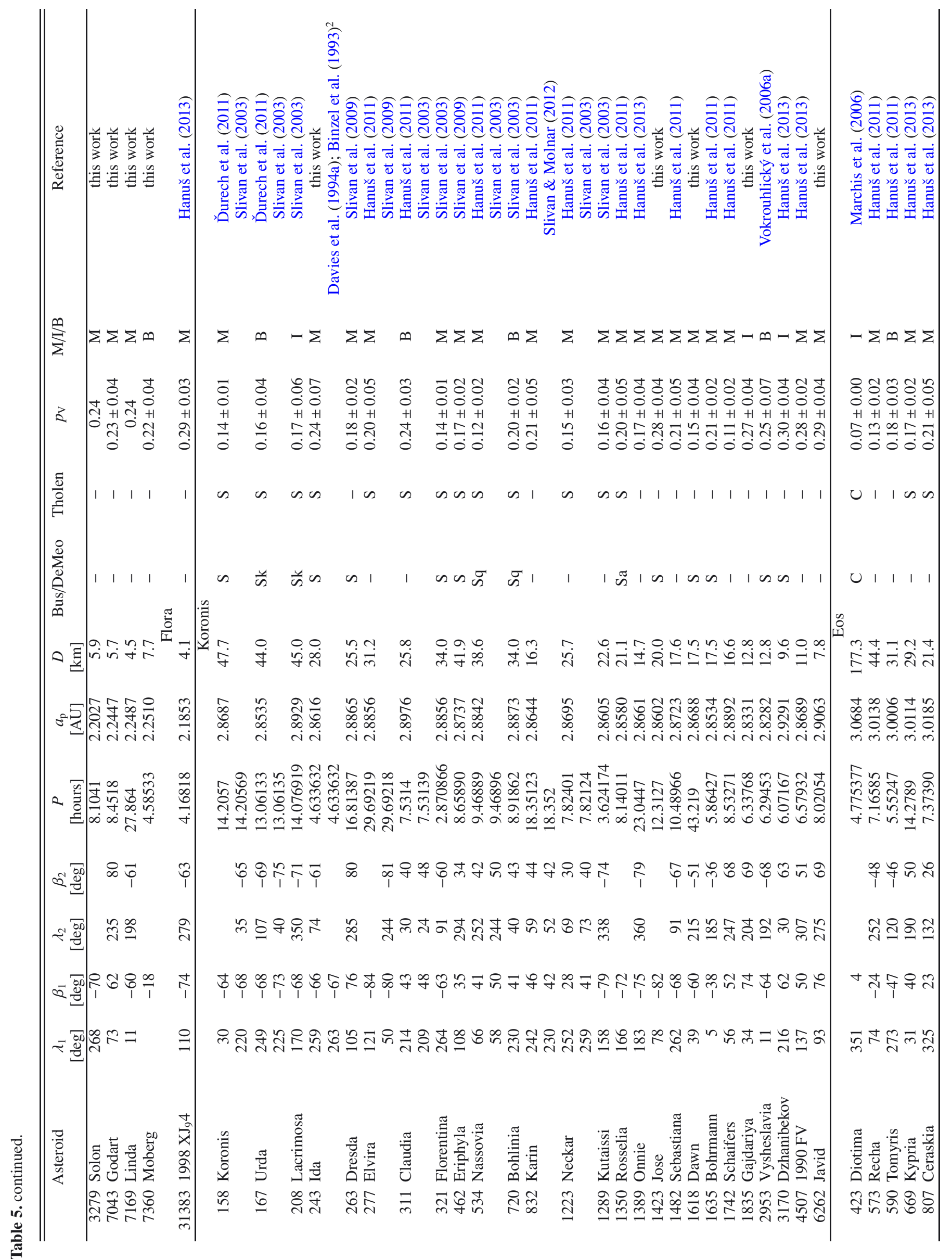




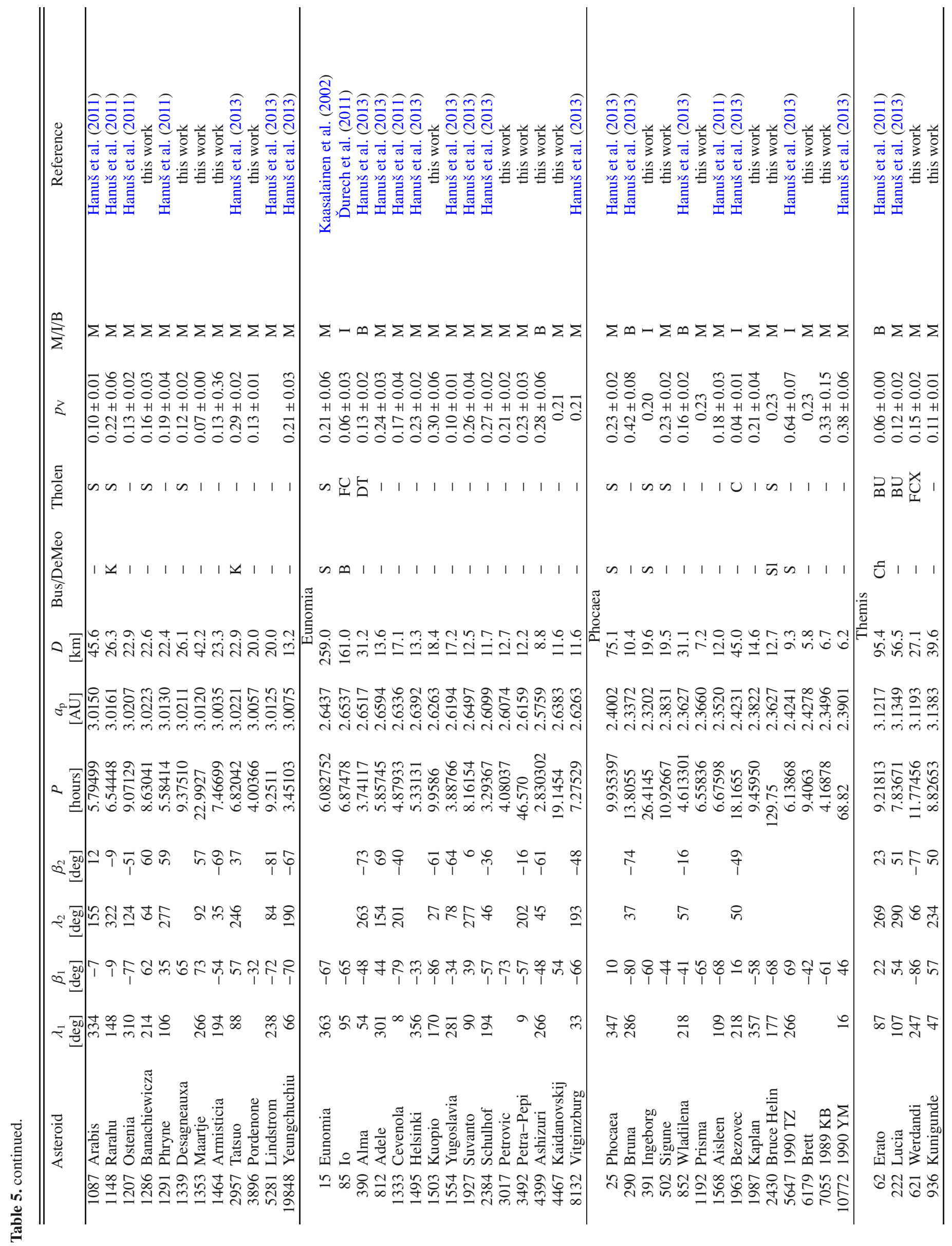


J. Hanuš et al.: Spin vectors in asteroid families

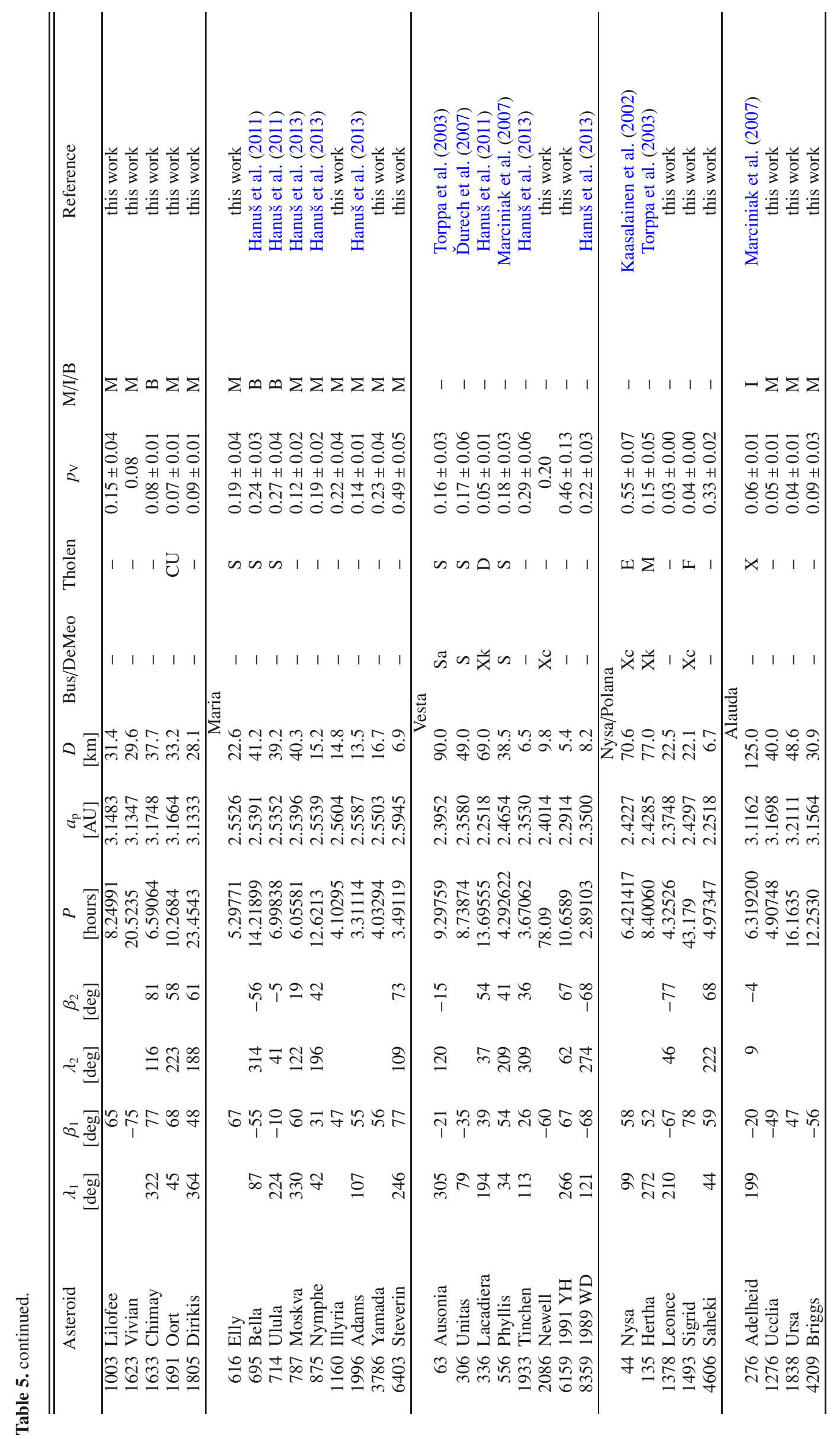

